\title{
SPHINGVM EVROPAEARVM
}

\section{ARVAE}

QVOTQVOT ADHVC INNOTVERVNT

$A D$

LINNAEORVM FABRICIORVM E T

VIEN NENSIVM INPRIMIS

CATALOGOS SYSTEMATICOS RECENSITAE

CVMTABVLIS AENEIS QVATVORDECIM.

ODER

DIE EVROPAEISCHEN

S C H W A E R M E R R A P E N

NACH DEN SYSTEMATISCHEN VERZEICHNISSEN

EINES LINNE, FABRICIVS

VND VORZUIGLICH

DER WIENERISCHEN NATVRFORSCHER

LATEINISCH VND TEVTSCH

IN VIERZEHEN ERLEVCHTETEN VND VNERLEVCHTETEN K VP FER N

AVFGESTELT

VON

HEINRICH WILHELM BERGSTRAESSER.

HANAV IM VERLAGE DES VERFASSERS, I78\% 
MVSAR/

\section{GA.}

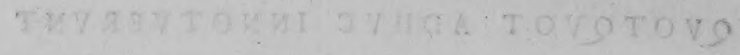

MVROHOIARAA WVAOIAVHIA T I I

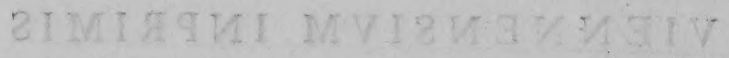

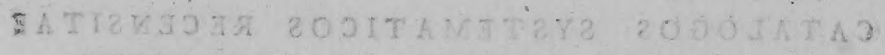

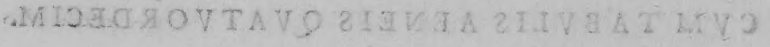

3000

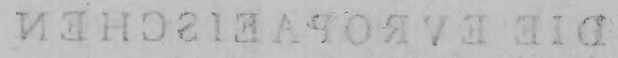

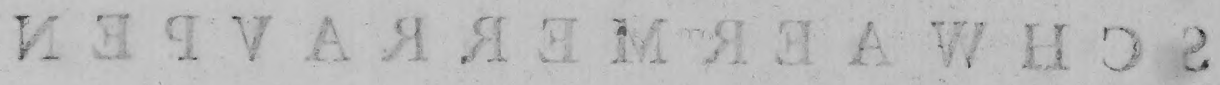

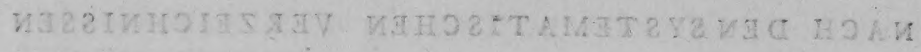

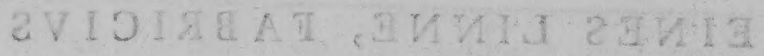

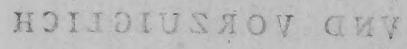

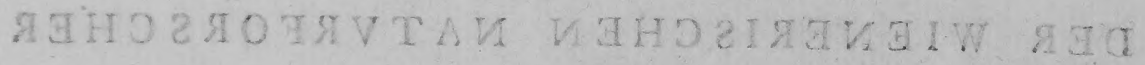

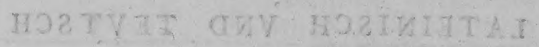

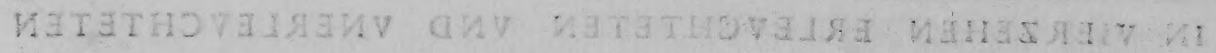
'ท मаяาม

TนลT2: อะVA

nov

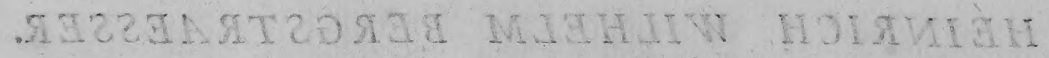

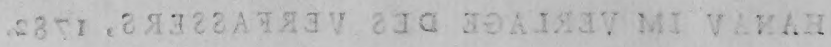


SPHINGVM LARVAE.

$S_{\text {phingum, quas dicunt, laruae }}$ omnes ve decem \& fex pedibus infrutae gradiuntur; ita earum pleraeque hinc cauda cornuue ad vndecimum corporis fegmentum, hinc laterum ftriis diftinguuntur, quarum quidem obliquitas a tergo verfus peEtus decurrit ; - in ceteris aut capitis corporisue figura dinerfae \& confpicuae, aut maculis, aut ocellis, aut pilofa cute, aut textili fub terram opere, ${ }_{2}$ quod pupas concludit.

\section{1.}

Sunt igitur acrocephalae aliae, aliae amblucephalae, aliae maculatae, aliae ophthalmicae; aliae elongatae, aliae fubpilofae, aliae, quod fuperat, phalaeniformes.

III.

\section{Schwarmerraupen}

\section{I.}

$\mathrm{D}$ ie Schwærmerraupen haber durchgehends fechszehn Fuife, fehr oft ein Horn auf dem Hinterleibe; oder dem eilften Ringe, nicht felten auch Seitenftriche, die fich von dem Horne und dem Ruikken im mer quer gegen die vordern Fuiff neigen.

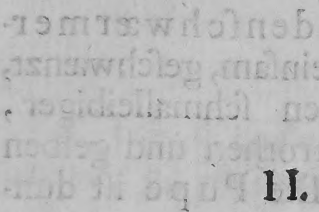

Sie theilen fich in Spizkopf. raupen, in Stumpfkopfraupen, in Flekkeraupen, in Augenraupen, in Langleibraupen, in Milchharraupen, in Scheinfpinnerraupen, A 2 
III.

Die Spizkopfraupen haben ein Horn auf dem Hinterleibe, einen oben zugefpizten fait dreiekkichren Kopf, eine blofe aber geriefelte Haut, bleichgelbe Querftriche an den Seiten. Die Verwandlung gefchieht unter der Erde ohne Geweb. Die Schwærmer haben eine fehr kurze zweitheilige Zunge und ausgezakte Fluigel.

1. Die Weidenfchwærmerraupe *) - einfam, gefchwænzt, geriefelt, gruin, mit fchrægen weisličhten Seitenftreifen, und zween gelblichten Augenpunkten.

2. Die Eichenfchwærmerfaupe **) - einfam, grï, fohrxgen und bleichgelben Seitentreifen, wovon einer um den andern breiter ift. Die Pupe ift ftumpf und kupferbraun.

3. Die Lindenfchwærmerraupe ***) - einfam, gefchwanzt, geriefelt, vornen fchmalleibiger, gruin, mit blutrothen und gelben Seitenftreifen. Die Pupe ift dunkelbraun,

ACROCEPHALAE ab capite, quod in trianguli acuti formam defcriptum eft, nomen habent erectoque ad anum cornu, cute fcabra, fed nuda, albidisque ad latera ftriis infignes funt. Puparum metamorphofin fine texto reconditæ fub terra fubeunt. Sphinges bifida lingtra, fed breuifima, alisque an. gulatis vtuntur.

3. Sph. ocellatae *) Larua folitaria, caudata, fcabra, viridis, Atriis obliquis lateralibus albis, punEtisque ocellaribus flauis. Pupa fufca, dorfo atro:

2. Sph. Quercus (roboris) **) L. - folitaria, viridis, ftriis obliquis pallidis alternis latioribus. Pupa obtufa, aenea.

\section{-8 vrituguib mit}

\section{-1000 1us o vilis}

3. Sph. Tilise ***) L $-\mathrm{fll}$ taria, cand ta, fcabra, antice attenuata, viridis, frriis lateralibus obliquis fanguineis, flauisque. Pupa obfeure fufca.

fik): Tab. 1. fig. T. Tab. 13. fig. 1. FABRIC, S.E. 536. 139. I. IINN. S.N. 2.

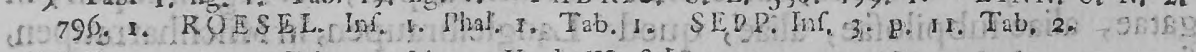
**) Tab. I. Gg, $x$. Tap. 13, Hig, 2. V. d.,W. Sch. p. 2+4:

F**) Tab. 2. fig 3. Tab. 3\%. fig. 3. FABRIC. S. E. 537. 139.4. LINN. S. N, 2.

$79 \%$ 3. ROESEL, Inf. 1. Phal, I. Tab, 2. 
4. Spib. Populi *4) L- Colitaria, caudata, fcabra, viridis vtrim. que linea ftriisque transuerfalibus, obliquis, albis. Pupa obfeure grifea, poftice ferruginea.

\section{LIII.}

AMBLOCEPHALAE as forma capitis obtufa dicuntur, quae quafi aequalieer vndique diffecta \& deprefla in ouatam fpeciem coit, angulo obtufiore. Nuda cutis eft. Cornu ad anum longius atterfa ex parte inflectitur, \& articulatim incurnefcit. Sub terra in pupam transfigurantur; at fine textilium munimentis. Sphinges integris \& acutis alis vt lancea fur: gunt \& quae naforum quafi iugo porreatae in pupis iam eminuerant linguae, earum nunc ingentes fipirasin orbem contuoluunt: quamquant eas facillime abdominis fimbriato alternis coloribus cinetu internof. cas.

1. Sph: Conuólusili*s) L caudata, verimque lineis lateralibus
4. Die Albernfchvexrmer. raupe * 4)- einfam, gefchviänzt, geriefelt, grtin, auf beiden Seiten mit einer blasgelben Linie in die Quere, und fchrægen Streifen! Die Pupe ift dunkelgrau und hinten rófterbig.

\section{IIII.}

Die Stumpfkopfraupen haben platte abgefehnitne eirunde Köpfe, eine blole : glatte Haut, ein langes krummes Horn auf dem Hinterleibe. Die Vervvandling gefchieht unter der Erde ohne Geveb. Die Schvværmer haben lanzetförmige unaussgezakte Flïgêf, ftarke Rolzungen, die fich meiftens durch eine nafenformigeErhoehung auch in der Pupe zeigen. Auf dem ganzen Leibe vvechreln zu beiden Seiten fchvvarze und helle Querftreife.

1. Die Windefchvarmer. ratpe *5) - ge(chrvenzt, nit fehra.

*4) Tab. 2, fig. 4. 6. Species valgaris. Fig. 4. a, Vaxietas. Tab, 13, fig, 4. FABRIC 5. E. 537. :39. 3. LINN.S. N. 2. 797. 2. ROESEL. Inf. 3. Tab. 30. SEPP. Inf. 3. P. 3. Tab. 8. fig. 5 .

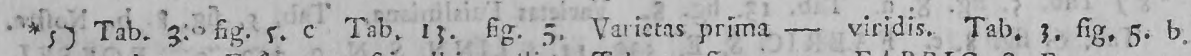
Secuxd - Fufe, punctis atbis nullis: Tab. 3. fig. 5, a. FABRIC, S. E. $544.2 \%$ LINN. S. N. 2. 798 , 6. ROESEL, Inf. 1. Phal, I. 'Tab. 7 . 
Schrægen Seitenlinien, und vveislichten augenformigen Punkten. Die Pupe ift helbraun und hat ein eingevvikeltes und zurükgebognes Horn.

2. Die Rainvveidenfchvværmerraupe *6) - gefchvvænzt, grün, mit fchrægen Seitenftreifen, die vornen fleifchfaerbig; hinten vveislicht find. Wenn fie ruht, fo hebt fie fich vorvvarts mit dem Kæerper in die Hoehe, und hælt die Klauen vor. Die Pupe ift braun und hat vier Zæhne am Schvvanze.

3. Dic Föhrenfchvværmerraupe *7) - gefchvvænzt, grünlicht, mit einer roffferbigen Rükken und gelben Seitenlinie, roffârbigen faft augenfermigen Luttlechern. Die Pupe ift helbraun.

4. Die Stechapfelfchyværmerraupe *8) - einfam, gefchwanzt obliquis, albis, punEtis fubocellatis. Pupa brunnea, cornu reflexo, involuto.

\section{Sph. Liguftri *6) L -} caudata, viridis, friis lateralibus obliquis, antice incarnatis, poftice albis. Quief́cit corpore antice ereEto, pedibus cleuatis. Pupa fufca, cauda quadridentata.
3. Sph. Pinaftri * 7) L. - caudata, virefcens, linea dorfali ferruginea, laterali flava, ftigmatibus, ferrugineis, fubocellaribus. Pupa brunnea,
4. Sph. Atropos *8) L. folitaria, caudata, flava, nigro punEtata.

*6) Tab. 4. fig. 6. Tab, 13. f. 6. FABR, S. E. 545. 28 . LINN. S. N. 2. 799. 8 ROESEL. Inf. T. 3. p, 25, Tab. 5. REAVM. Inf, 2, Tab. 20, fig. I-4. :SWAM. MERDAM. Bibl. nat, Tab. 29. fig. 1, 2. 5. SEPP. Inf. 3. P. I9. Tab, 4. DEGEER. Inf. 1. Tab. 1, fig. 6. HARRIS. Inf. Tab. 2, fig. a-f.

7) Tab. 4. fig. 7. Tab. 13. fig. 7. FABRIC. S. E. 54I, 16. LINN, S. N. 2. 802, 22. ROESEL, Inf. 1. Phal, 1. Tab, 6. SEPP. Inf, 3. p. 23. Tab, 5. DEGEER. Inf. I. Tab, 10, fig. I, 2, 3.

* ) Tab. 5. fig. 8. a. Tab, 13. fig. 8. Varietas Fuisliniana, Tab. 5, fig. 2. b. Noftu.
Tab. 6. fig. 8. c. F A BR IC. S. E. Tab. 6. fig. 8. c. F A BRIC. S. E. 539.1 I, LIN N, S. N. 2. 799. 9, ROESEL,
3. Tab, 1, 2, FVESLIN, Archiu. erf. Heft, 
Etata, lineis lateralibus transuerfis dimidiato caeruleis viridibusque, cauda deflexa. Puppa brunnea, ftig. matibus vtrimque quinque nigris.

MACVLATAE minora \& anguftiora habent pro corporis portione capita, eaque globofa \& por recta. Cutis nuda \& laevis eft. Cornu ad anum recuruum. Maculae in lateribus pallidae. Puparum metamorphofin in terra opperiuntur, contexendo ante pofitas exuuias foliorum inuolucro. Sphingum, quas in orbem torquent, linguae anguftiores funt. Alae oblongulae, integrae. Fafciae veringue nigrae dimidiatas abdominis cinetulras ambiunt.

\section{Sph. Euphorbiae *9) L. -} caudata, nigra, albo punctata, linea dorfali languinea, laterali punEtisque flauicantibus. Pupa brun. nea ftigmatibus nigris, fchvvanzt, fchvvarz punktirt, mit zur Hælfte blanen, zur Hælfte grû. nen Seitenftreifen in der Quere, und herabgebognen Schvvanze. Die Pupe ift helbraun und hat auf beiden Seiten fünf fchvvarze Luftlo-? cher.

\section{V.}

Die Flekkeraupen haben kleine kuglichte Kœepfe, die fich nicht einziehen, eine blofe glatre: Haut, ein Horn auf dem Hinterleibe, durchans bleiche Seitenmakeln. Die Vervvandlung gefchieht auf der. Erde mit niber fich gefponnenen Blattern. Die Schvvarmer haben fchmale Rolzungen, langlichte un ausgezakte Fluigel, nur auf dem halben Leibe an beiden Seiten fchvvarze Querftreife.

\section{Die Wolfsmilchfchvvær.} merraupe *9) - gefchvvænzt, fchvvarz, vveislicht punktirt, mit einer blutrothen Linie auf dem Ruik. ken, und einer gelblichten auf den Seiten, mit gleichfarbigen Punkten. Die Pupe ift helbraun und hat fchvvarze Luftloecher.

2.

$$
\text { z. }
$$

9) Tab. 6. fig. 9. Tab. 13. fig. 9. FABR IC. S. E. 54T. 17. LINN. S. N. 2802 19. REAVM Inf. I. Tab. 13. fig. 1. 4. 5. 6. ROESEL, Inf. I. Phal. I. Tab. DEGEER, Inf. r. Tab. 8. fig. $6-11$. 
2. Die Walfrohfohver: merratipe *10) - ge(chvvænzt gruin, mit einer gelben Ruikkenlinie, und gleichfarbigen Guirtel. freifen an dem Ende der Ringe gegen die Einfchnitte, nebft derglei: chen Flekken auf den Seiten. Die Pupe ift vornen dunkelbraun und hinten blas.

- ge(chvvenzt* ${ }_{\text {Il }}$ ) fehvvarzmit einer gelben Ruikkenlinie, gleich-. frerbigen Guirteln an den beiden Finfchnitten der Ringe und eben folchen Flekken an deriSeiten. Erfte Verfchiedenheit.

- ge(chvvænzt, * 12) gruin, und nur blos durch die gelben runden. Flekken auf den Seiten ausgezeich. net. Zvyeit e Verfchiedenheit.

3. Die "Kcechlinsfchvv ærmerraupe *13) - gefchvvanzt, fchvvarz, miteiner blasrothen Ruikkenlinie und głeichferbigen runden Flekken an den Seiten. Die Pupe ift helbraun, und hat fohvvarze Lufulcecher.

\section{VI.}

2. Sple, Galit *10) I. caudata, viridis, linea dorfali, cinฯ gulis ad extremas fegmentorum incifuras, macularumque in lateribus. rotunditate flauis. Pupa antice fufca; poftice pallida.

- catudata, * I I) nigra, linea dor. fal, cingulis ad vtrasque fegment. torum incifuras, maculifgue in la teribus flaus. Varietas prima.

- caudata, * I 2) viridis, fola flauarum in lateribus macularum ro-; tundarione diftincta. Varietas fecunda.

3. Sph. Koechliniance* 1,3) L. caudata, nigra, linea dorfali, quaeque ad latera in circinos rotundantur, maculis pallide rufis, Iupa brunnea, fligmatibus nigris.

VI.

* 10) Tab. 6. fig. 10. Tab, 13 . fig. 10. ROESEL,

*11 ) Tab, 7, fig, 10, b.

*12) Tab. 7, fig. 10, c.

* 13) Tak. 7. fig. II. Tab, 14. thg. IT. FVES L. Architi, erft. Heft, 


\section{VI.}

OPHTHALMICAE fphingum laruae ab oculeis latcrum in collo maculis nomen trabune, tantum abeft, vt ab vifus acie, quod opineris, appellentur, quippe qua nullum erucarum genus penitus ca. rere coniftet. Exile omnes \& in orbis fpeciem globatum caput facili profer molles commiffuras porreetione lanceant, aut in proximorum fegmentorum tumores tanquam in vaginam recraktu condunt. Caudas habent. Cutis \& pilorum expers \& foabritiarum, nuda eft. $M e$ tamorphofin maculatarum fimilem in terra habent, textorio fub foliorum integumento lacitantes. In jphingibus linguarum firiae minores funt. Alae oblongae. Poft: cus incernusque margo arcuata ver. fus batin cautitate in obliquum recedunt. Anus cufpidatim defcendit,

r. Sph. Nerii * 14) L. - foli. taria; catdata; albo punktaca, collo ocello yerimque coeruleo, cauda deflexa, fubarticulata. Pupa flaua, linea dorfali puntisque lateralibus vigris.

\section{2. $\mathrm{B}$

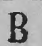

I. Die Lorber rofenfchwarmerraupe * 14) - einfam, gefchwænzt, weis punktirt, ein blaues Aug auf beiden Seiten am Halfe, mit gebognen und begliederten Schwanze. Die Pupe ift gelb, hat eine fchwarze Linie auf dem Ruikken und dergleichen Punkte auf den Seiren.

2.

* 14) Tab. 8. Tab, 14, fig. 12, FABRIC, S. E. \{38. 5. LINN, S, N, 2, 798.5 ROESEL, InS, 3, T, 16. 
2. Die Weintokfchvvarmerraupe * 15 ) - gefchvvenzt, dunkelbraun, mit zvvoen vveifen Seitenlinien, und zvveien Augen am Halfe. Die Pupe ift vorn dunkel; hinten helbraun.

3. Die Schotrenvveiderich(chvvarmerraupe *16) - geGhvvanzt, dunkelbraun, geflekt, mit zvveien blauen Augen am Halfe aut den beiden Seiten.

- dunkelbraun, * 17) mit zvvoen veifen Seitenlinien und dergleichen Querftreifen, erfte Verf chiedenheit.

- gruin *18). Zweice Verfchie. denheit. Die Pupe ift vorn dunkelgrau; hinten braun; die Luftleecher find dunkler geforbt.

4. Die Labkraut fchvvermerraupe * 19)- ungefchwanzt, dunkelbraun, mit drei blauen Augen am Halle auf jeder Seite. Die Pupe ift fchwarzlicht.

- gruin. *20, Erfte Vcrfchiedenheit.

Die Langleibraupen - haben einen kuglichten Kopf, einen langen cylindrifchen Leib ohne Augen-
2. Sph. Celerionis * I5) L. caudata, fufca, lineis duabus lateralibus albis ocellisque vtrimque duobus collaribus. Pupa antice fufca, poftice brunnea.

3. Sph. Elpenoris * I6) L. caudata, fufca, maculata, ocellis collaribus vtrimque duobus coeruleis. Pupa antice obfure grifea, poftice fufca, ftigmatibus obfcurioribus.

- fufca * 17) lineis duabus lateralibus, Atriisque albis, Varietas prima.

viridis. * I8) Varietas altera.

4. Sph. Porcelli *19) $\mathrm{L}_{+}-$ ecaudata, fufca, ocellis vtrimque tribus collaribus coeruleis. Pupa nigricans.

\section{- viridis*20). Varietas prima. VII.}

ELONGATAE a cylindracea corporis fructura \& longitudine dictae funt. Caput in rotundita-

15) Tab. 9. fig. 13. Tab. 14. fig. 13. FABRIC. S. E. 545. 30. LIN N. S. N, 2. 800. 12. ROESEL.

* I6) Tab. 9. f. I4. b. Tab. I4. f, 14. FABRIC. S. E, 543.25. IINN. S, N, 2. 8OI. 17. ROESEL. Inf. I. Whal. I. Tab. 4.

* 17) Tab. 10, f, I4, c.

* 18) Tab. 9. f. 14. a. Tab. 10. fig. 15. a. Tab. 14. fig. 15. FABRIC. S. E. 544. 26. LINN, S, N. 2.

801. 18. ROESEL. Inf, I. phal, I-5.

* 20) Tab, I0. f. Is. b. 
tatem globatur. Colli ozuleis ma- genmakeln an den Seiten, ein Horn culis carent, \& pro cauda, In qui• bus deef, ad anum circulum oculatum gerunt. Fit earum transfigurutio in terra eadem, quin ophthalmicarum, ratione, nifi quod praeter foliorum, fi in prometu funt, graminum quoque textu obtegantur. Sphingum lingra fpiras conuoluunt easque longiticulas. Alae fere bre. uiores; corpora latiora funt \& craffiora. Barbulas ad lutera \& in abdomine fubmittunt. Volatu vel diurno gaudent.

I. Sph. Oenlotherae * 2r) L. ecaudata, furdide fulca in dorfo, ad latera pallida, ftigmatibus anique ocello coeruleis. Pupa brunnea.

2. Sph. Stellatarum * 22) L.caudata, e flauo viridis, albo punctata, cauda fubulata, coerulca, apice ferruginea. Pupa fufca.

\section{VIII.}

SVBPILOSAE a pilorum in cute albefcentium teneritate \& pube funt appellatae. Caput ab antica parte feu dimidia in orbisfpeciem globant. Cornu ad anum in curuefcit. Pupam filamenti vndivndi- oder antat deffelben einen Spiegelflekken auf dem Hinterleibe. Die Verwandlung gefchieht auf der Erde, mit uiber fich gefponnenem Grafe, oder Blættern. Die Schwærmer haben lange Rolzungen und fatt kurze Fluigel, find an den Seiten und am Ende des breitgedruikten Leibes barticht, fliegen auck beim Tage.

r. Die Nachtkerzenfchwærmerraupe - ungefchwinzt, fchmuzigbraun auf dem Ruikken, an den Seiten blasgelb, mit Luftlcechern, die gleich dem Augenfpiegel auf dem Hinterleibe blau find. Die Pupe ift helbraun.

2. Die Sternkrautfchwærmerraupe - gefchwænzt, gelblicht grthil, weis getuipfelt, mit einem privenenfermigen Horne, das unten blau und an derSpize roftfxr. big ift- Eine dunkelbraune Pupe. VIII.

Die Milchharraupen haber, fo viel davon bekant ift, einen halbluglichten Kopf, einige auch ein Horn auf dem Hinterleibe, Die Haut ift mit fehr feinen weislichten Haren befezt. Die Verwandlung B 2 ge-

*21) Tab, II, f. 16. Tab, 14, f, 16.

*22) Tab, 11. f. 17. a. T. 14. f. 17. FABRIC. S, E, 548. 3, LINN, S, N. 2. 27. 803. ROESEL, I, phal, I, Tab. 8. 


\section{2}

gefchiehc in einem Gevvebe. Die Schwärmer haben feine Rolzungen, am Ende des Leibes insgemein ein breites Harbuifchchen, glasartig durchfichige Flïgel, fliegen auch in der Sonne.

I. Die Skabiofenfchwärmerra upe - gefchwänzt, grïn, mit einer w'eislichten Seitenlinis, die vom helbraunen Horne an, nach der Bruft zulaufr. Die Luft. löcher und der untere Siand find holbraun gefäibt

2. Die Pappelbraumfchwärmerraupe - gefchwänzt, griiu, mit zween holbraunen Fleken auf iedem Ringe.

$$
\text { VIIII. }
$$

Die Scheinfpinnerraupen find träg, haricht, gew ölbt meiftens mit zwoen oder vier Reihen fchwarzer Punkte, mit einem kleinen verdekten Kopfe, und verdekten Füffen. Die Vervvandlung gefchiehs uber der Erde in einer glänzenden ge. vö̈lbten Höle, Die Schwärmer haben Rolzungen, fchvvarze, faft gekolbte Fuihl hörner und lange abhaengende Flïgel mit hochfärbichten Flckchen, fliegen nur bei Tage.

1. Die Erdreichelfchwärmerra.upe - dikleibig, fchvvefelgelb, mit vier Reihen fchwarzer Punkte. Die Pupe in dunkelbraun, in der Mitce Ichvvefelgelblicht, die Lufülöher dunkelbraun.

* 23) Tab, 12, fig. 18. t. 14. f. 18. a, b. F ABR. S. E. 5+8. 5, LINN, S. N, 2, 803, $28^{\circ}$ ROESEL. 4. tab. 34. f. $1-4$.

* 24) Tab. 12. f. 19. T. I4. f. 19. FABRIC. T. E. 548. 5. ROESEL. In1. 3.38.

- 25) Tab. 12. f. 20 t. I4. f. 20. a. b. FABRIC. V. E. S5O. I. LINN. S, N. 2. 805. 34. ROESEL. Inf, 1. phal, 2, tab. 57. REAYM. Inf. 1. tab. 12, fig, 14 - 17. Lif, 2, tab, 2, fig, 2 . que texto concludunt. Angunas. fphinges linguarum fpiras habene, $\&$ in extremo abdomine comatam barbulam. Alae vitri inftar pelluccnt. Apricantur \& in fole volant. 1. Sph. Fuciformis * 23) L. caudata, viridis, linca laterali a cau. dabrunnea; rerfus peetus albida, ftigmatibus \& margine brunneis.

\section{Sph. Cralroniformis * 24) L.} - caudata, viridis, fegmentis verimque maculis duabus brunneis.

\section{VIIIJ.}

PHALAENIFORNES Obefa \& pilofa conuexitate furgunt, pluribusque nigiorum punetorum ordinibus notantur. Caput, quod fane quam exiguom ent; obnubitur. Pedes operti funt. Sub terram transformantur textoria vndique convexitate, eaque fplendida conclufae. Sphinges linguas fpiris exfertas \& fetaceas labent, antennasque nigras, in extremitatibus quafi clauatas. Alae rendulae \& pro corporis mole longiores macularum claritate colorumque exfplendefcunt. Diurnae volitant.

1. Sph. Filipendulae *25) L. - obela, fulphurea, lineis quatuor punctorum nigrorum. Fupa fufca, medio fulphureo, ftigmatibus fufcis. 


$$
\mathbb{*}
$$




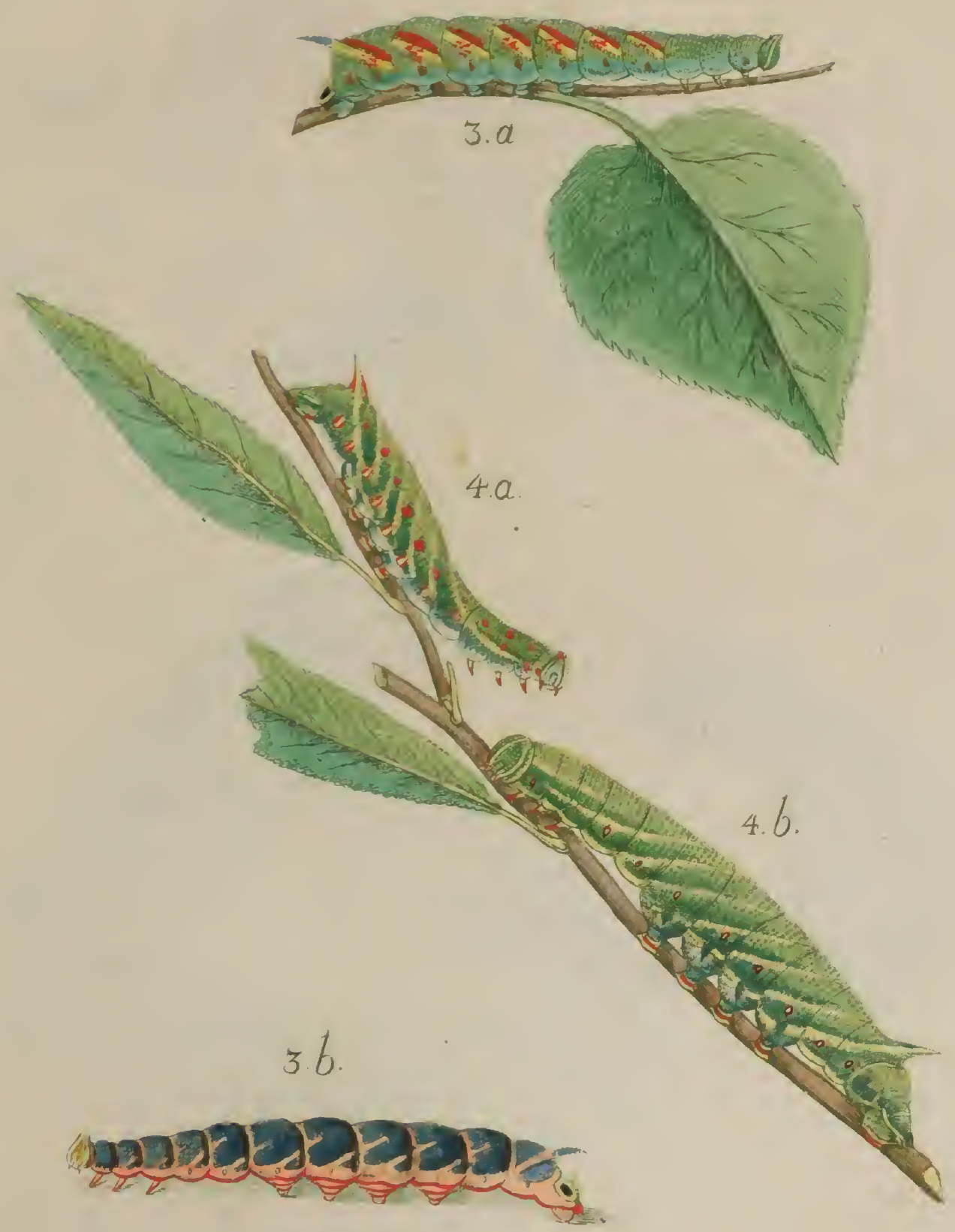




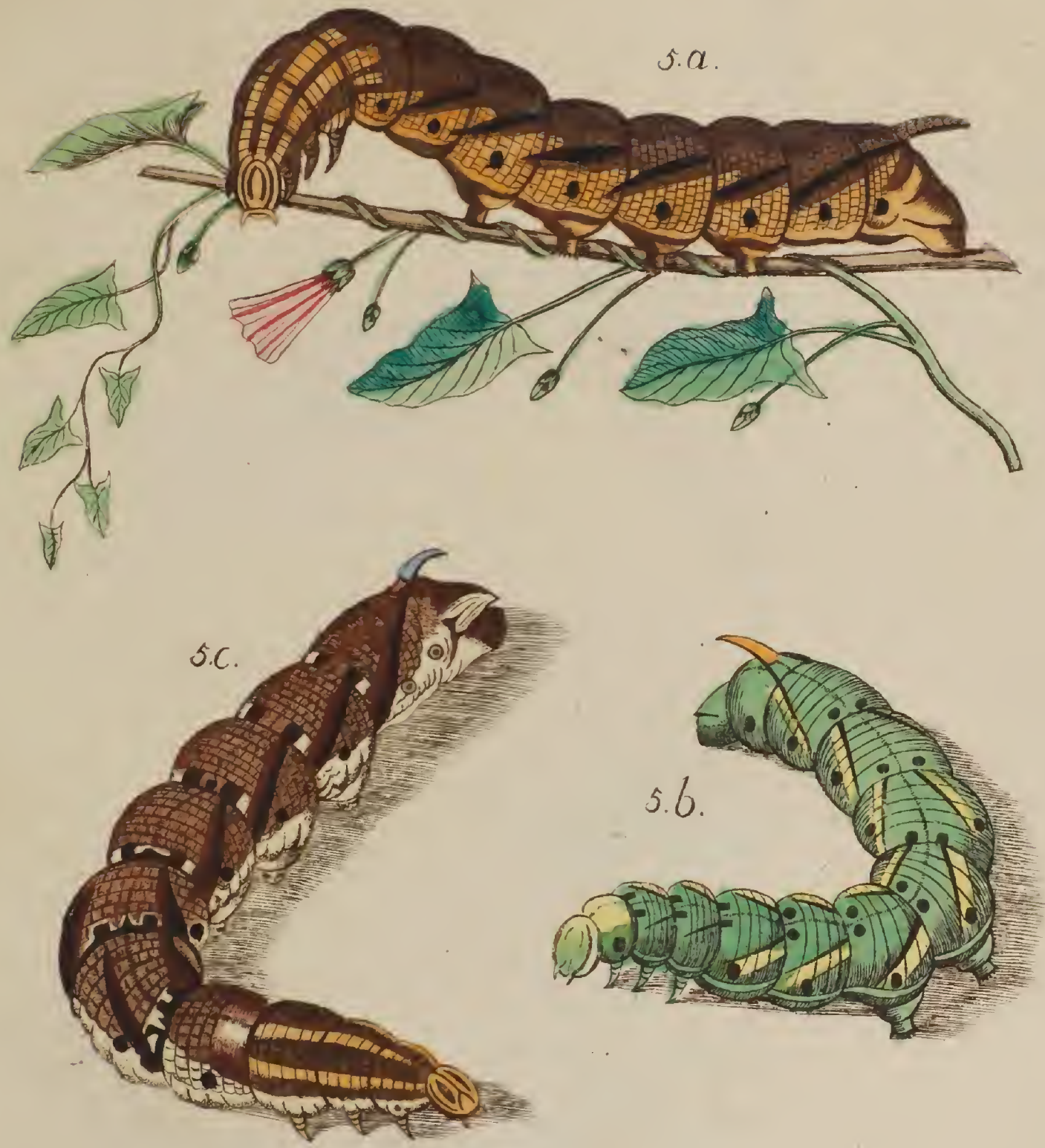




$$
\frac{1}{2}
$$



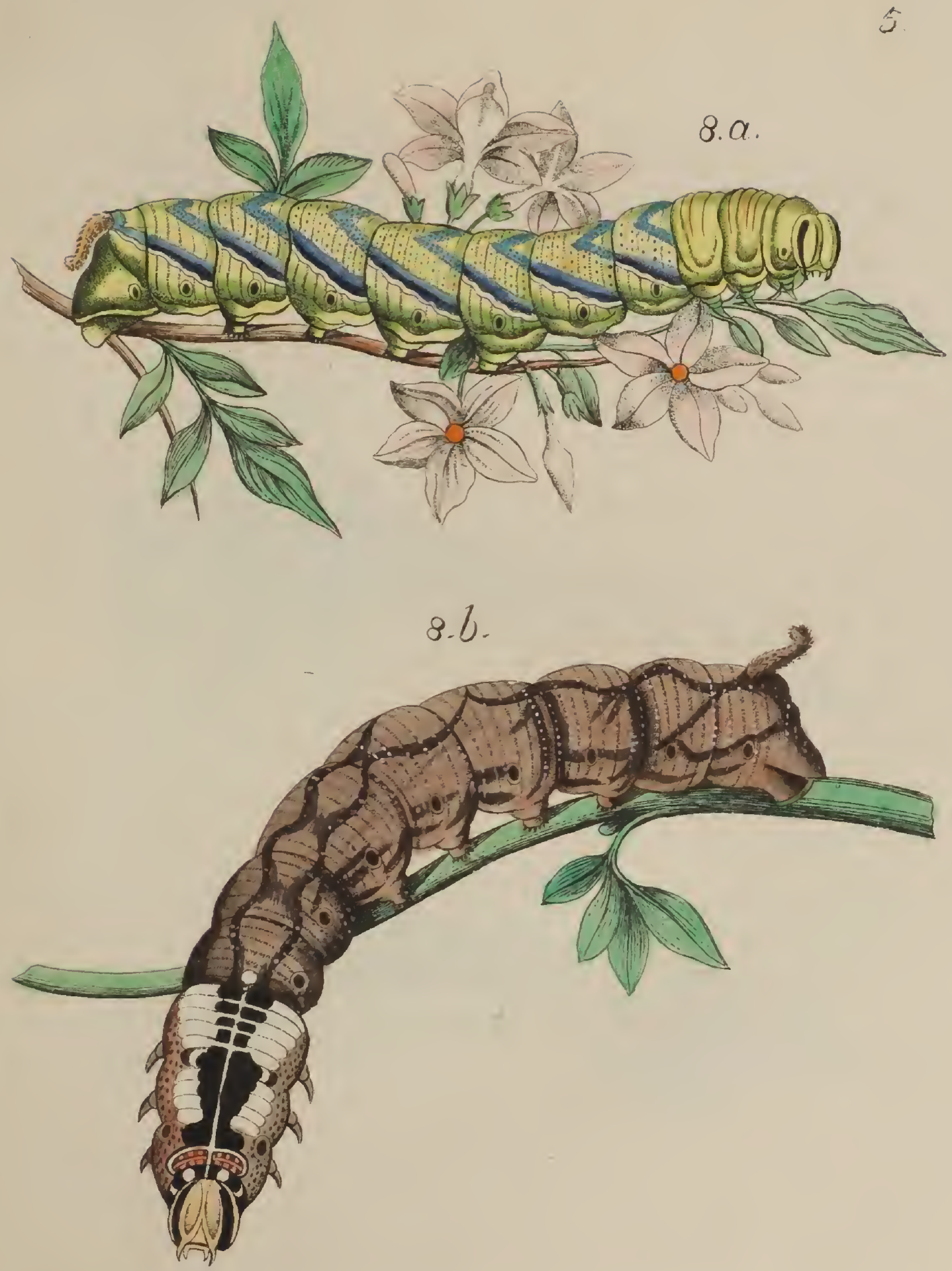
6.

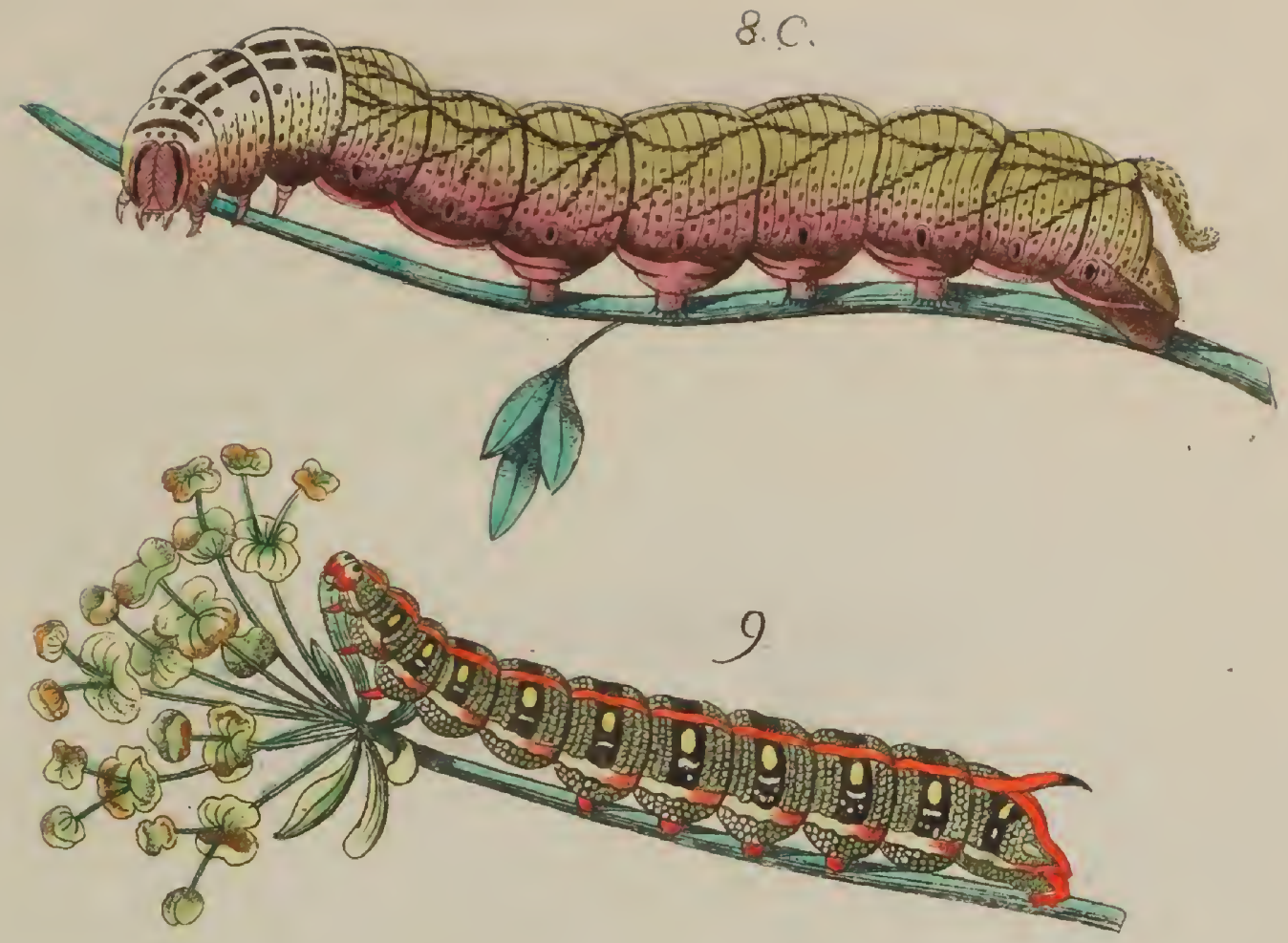

10. $a$.

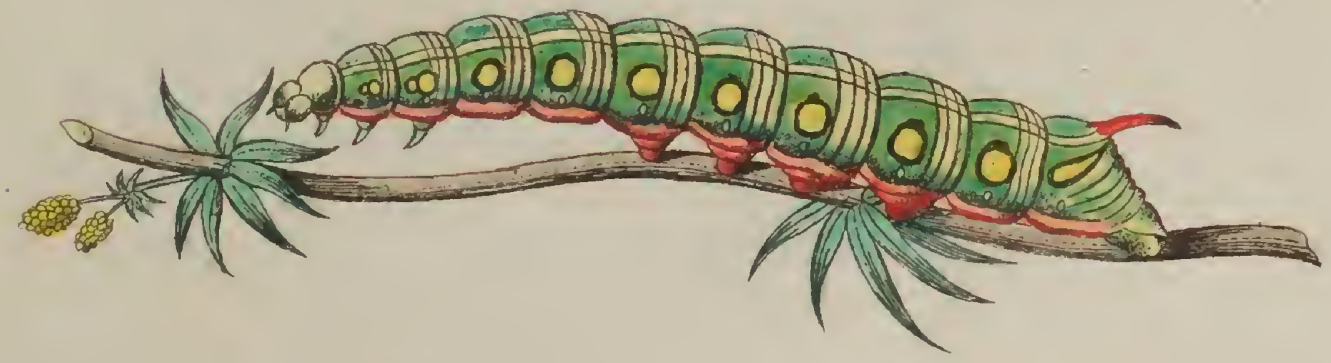




\title{
10. $b$.
}

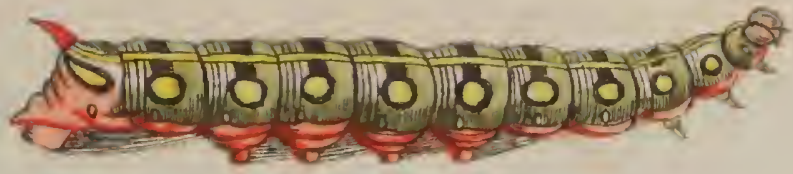

\author{
10. C.
}
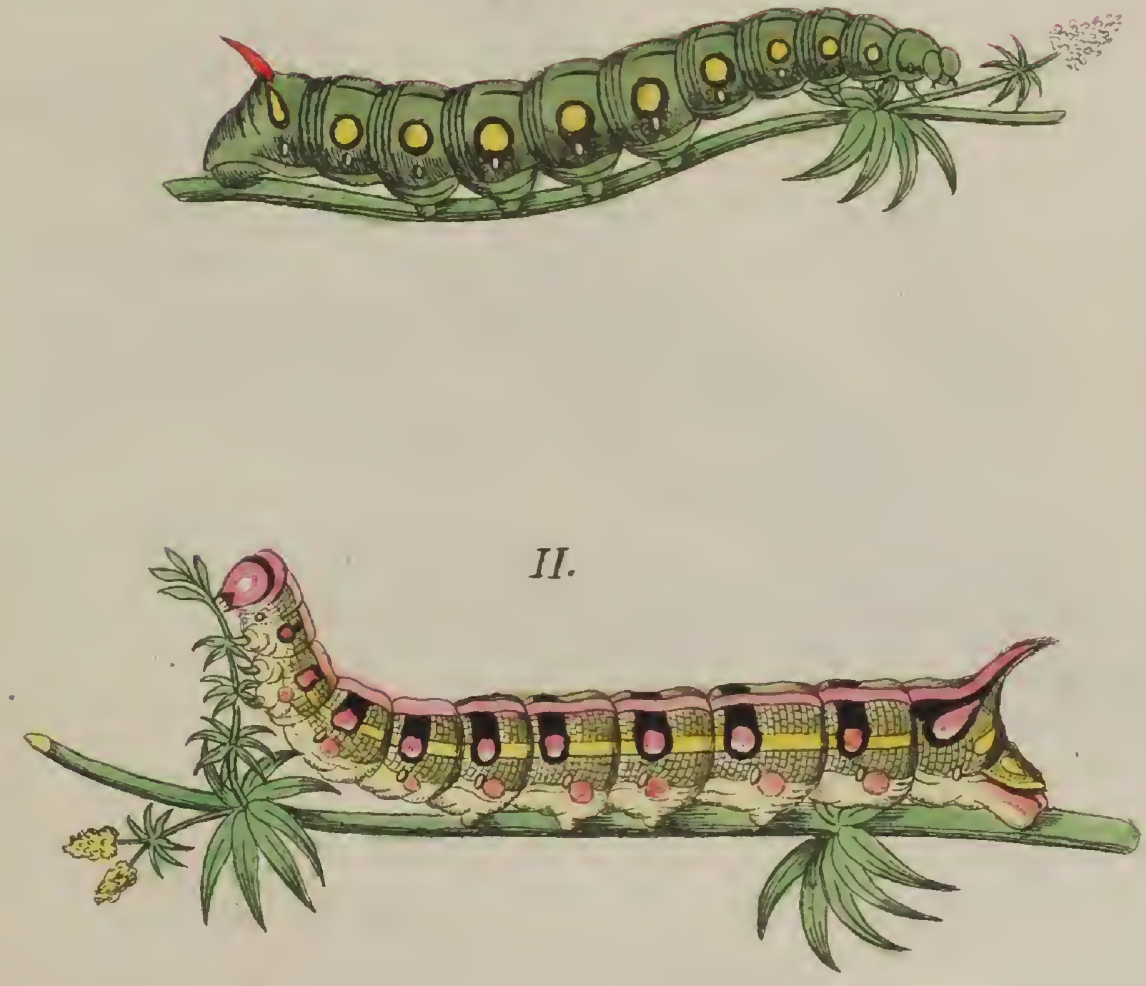


\section{2. $a$.}

arvinger

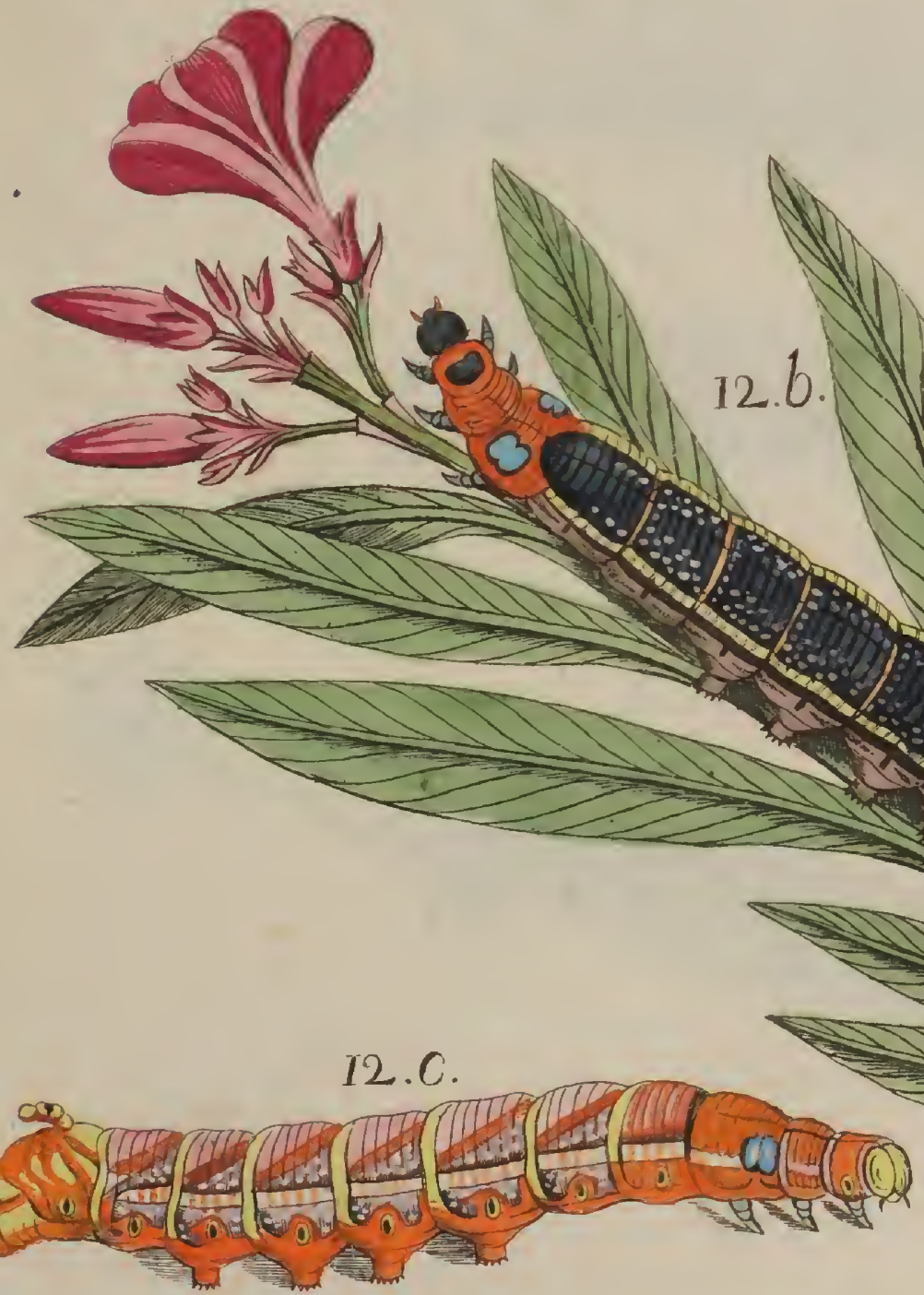



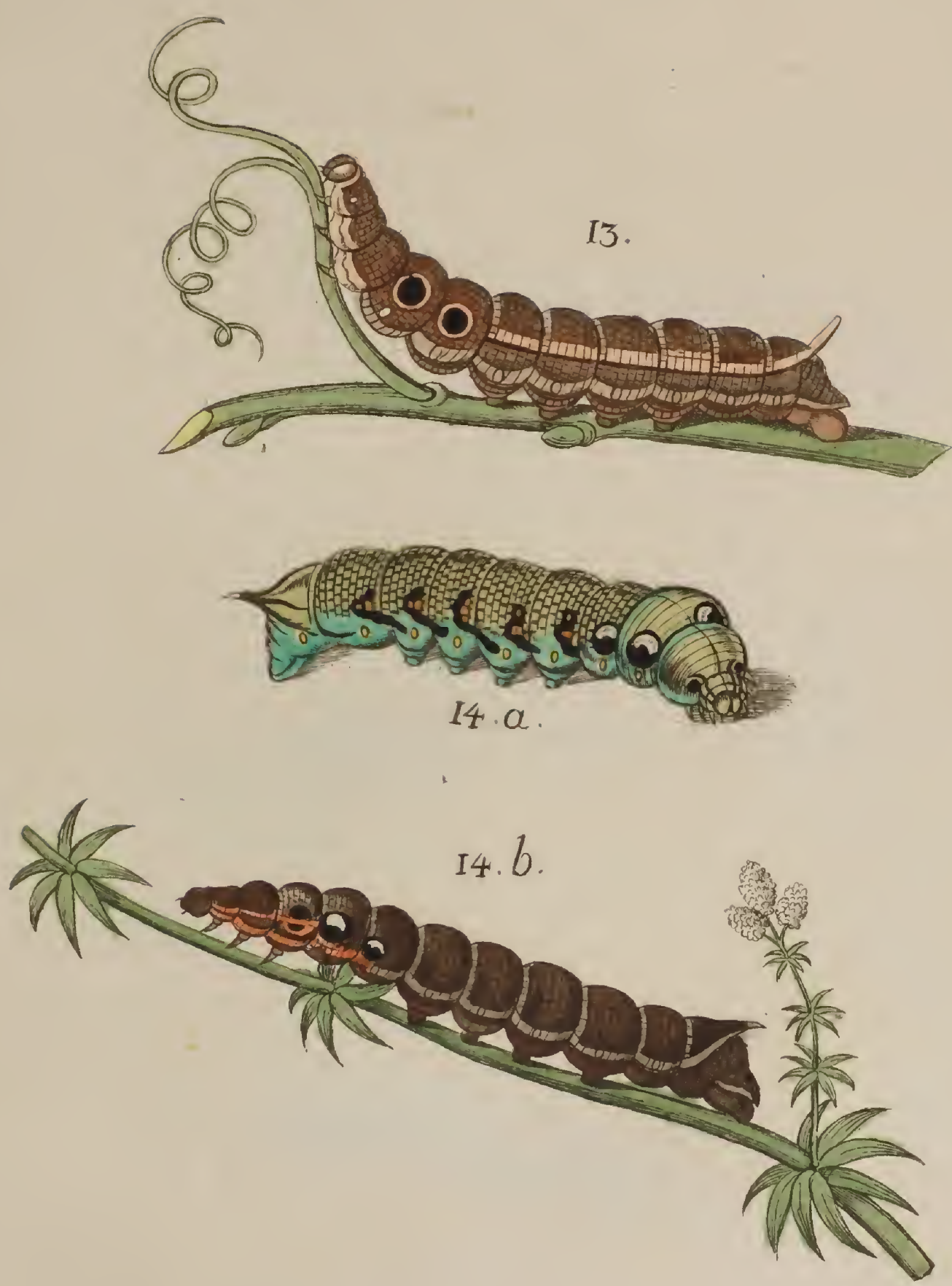
IO.
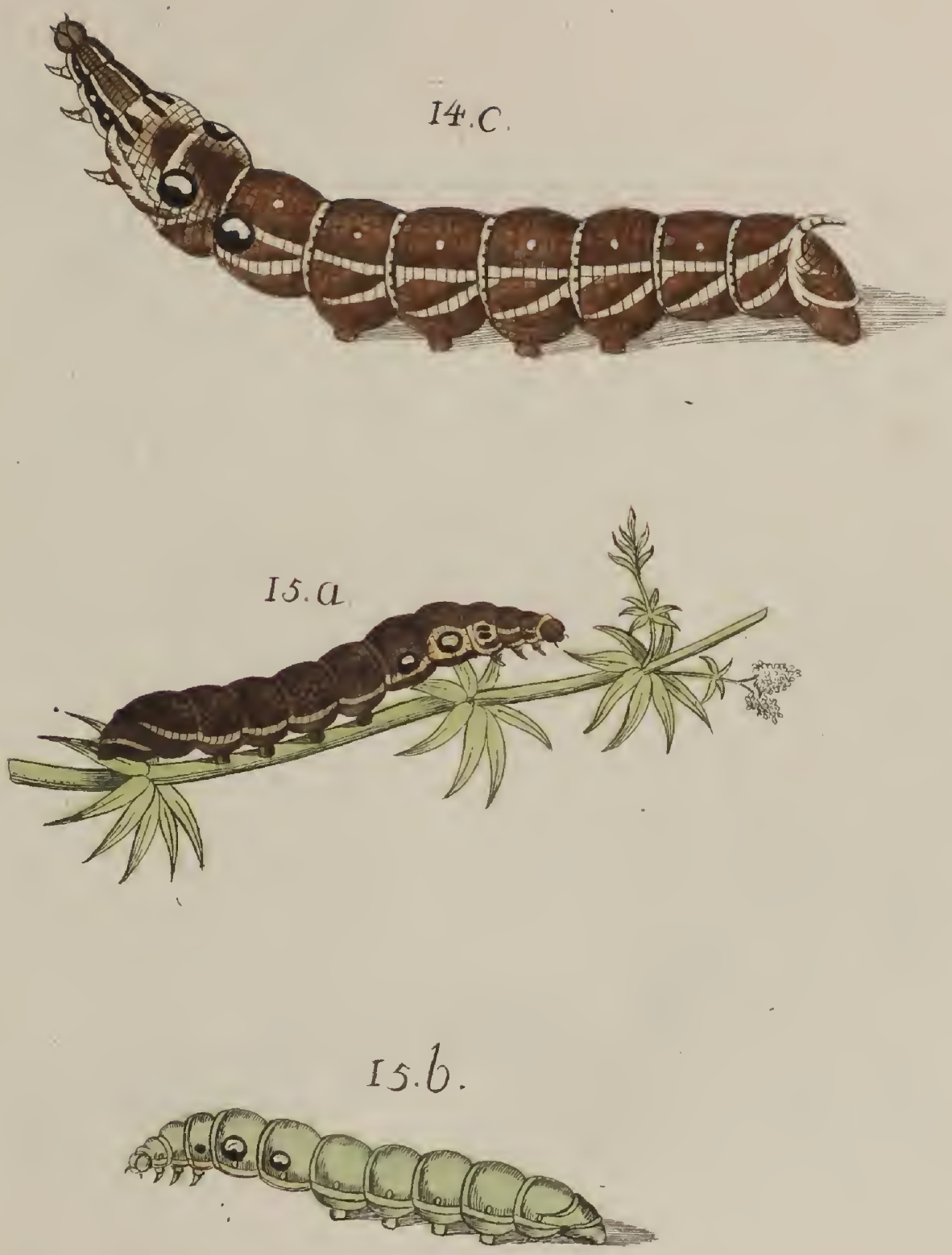


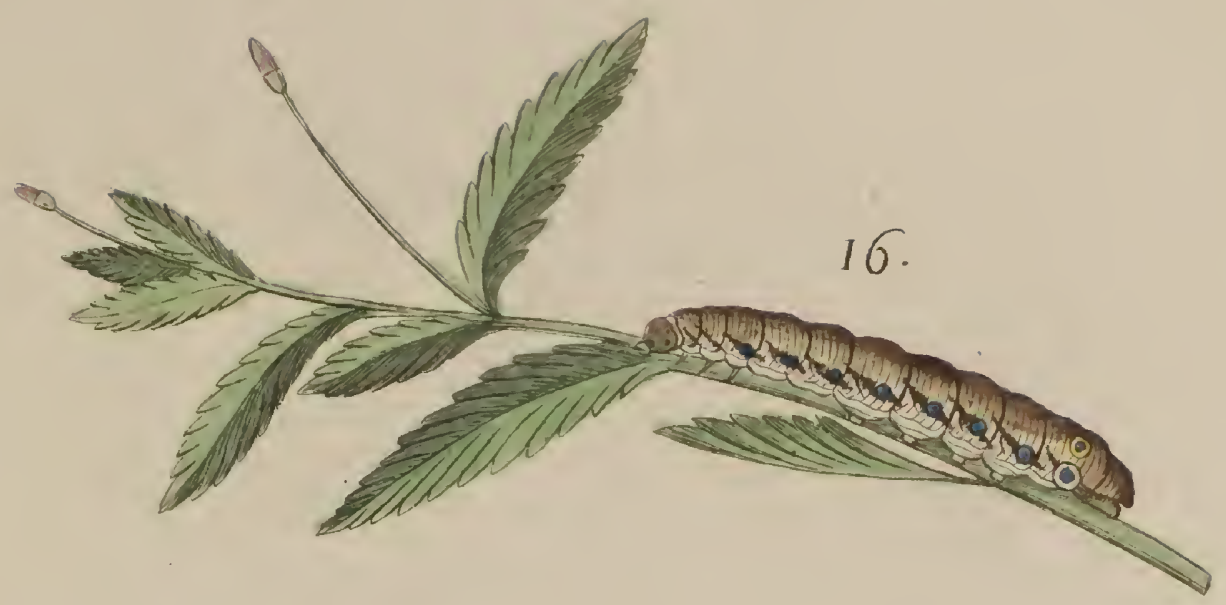

17. a.

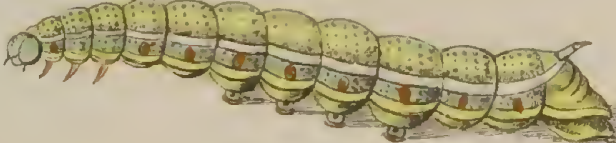

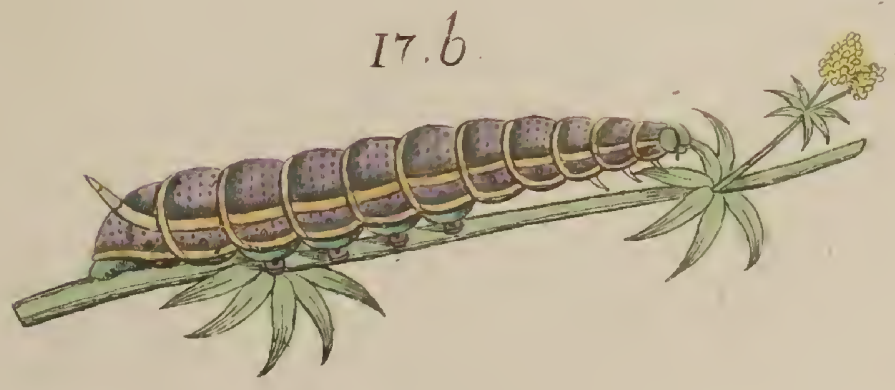



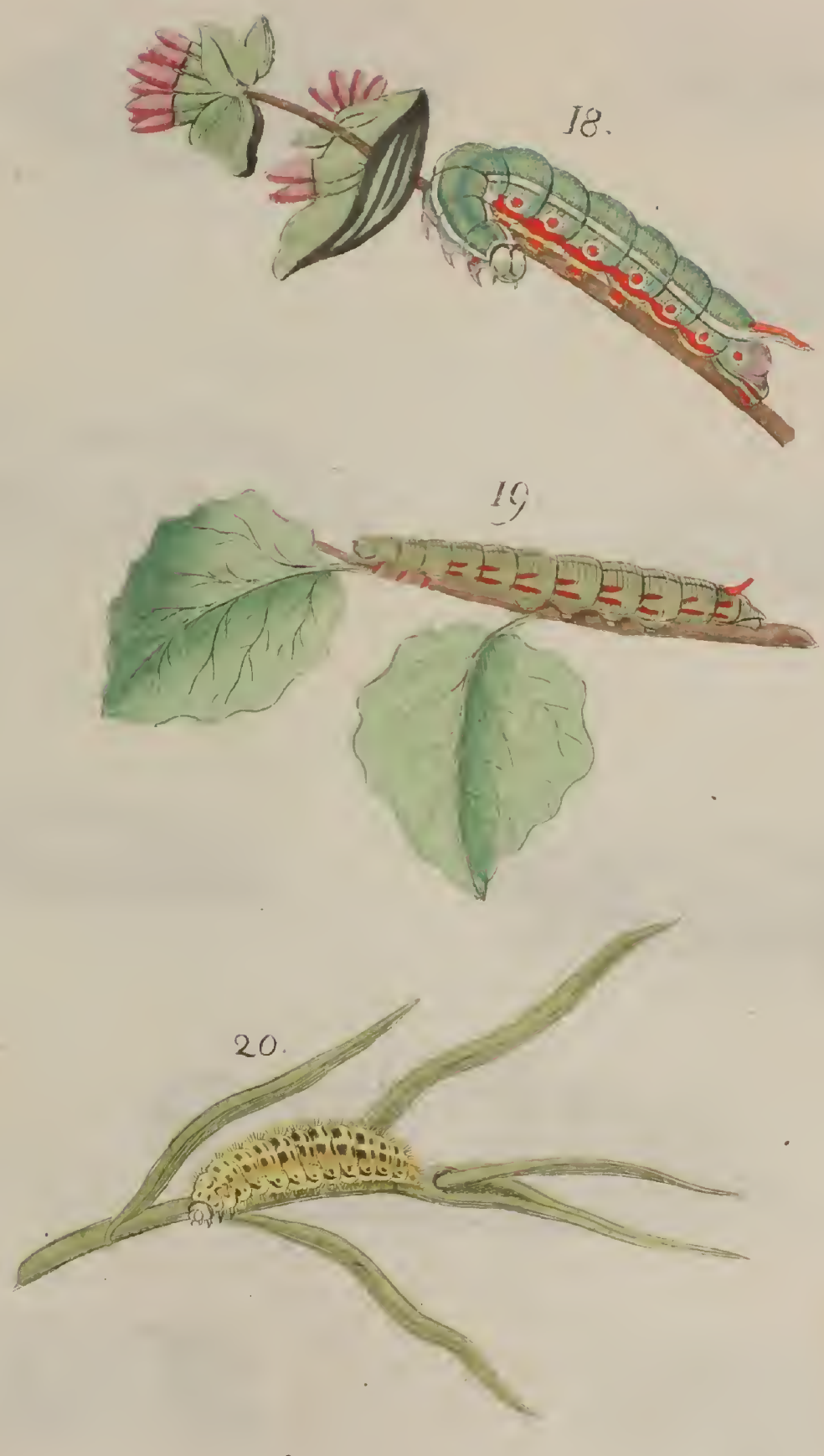


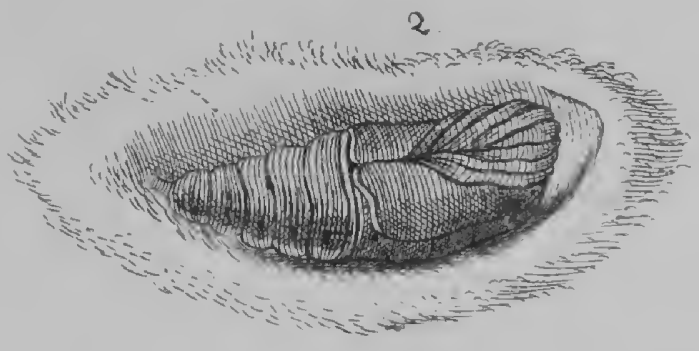

5
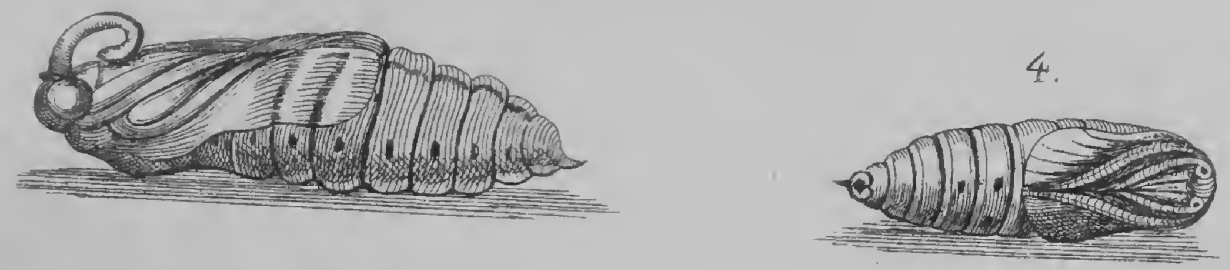

6

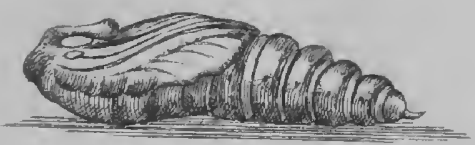

4.

$I$.

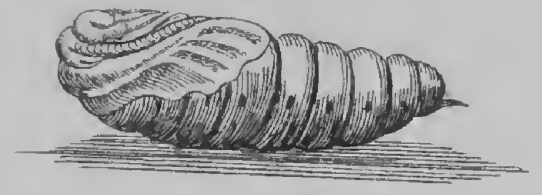

3

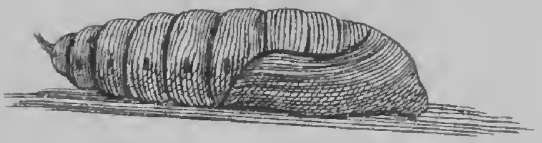

4.

8.

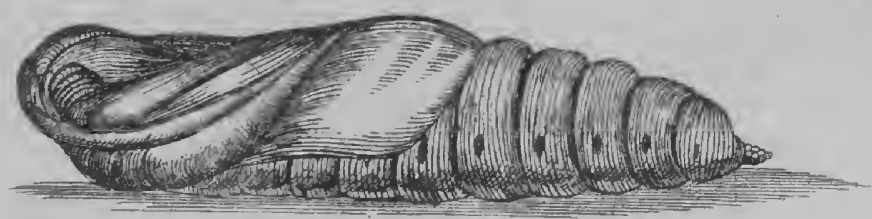

10

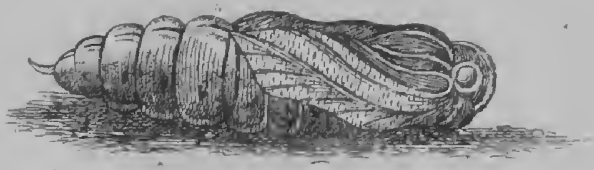




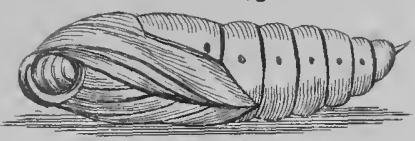

14.

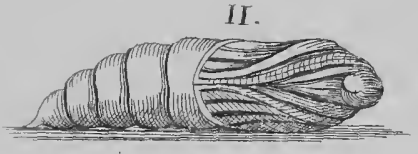

12

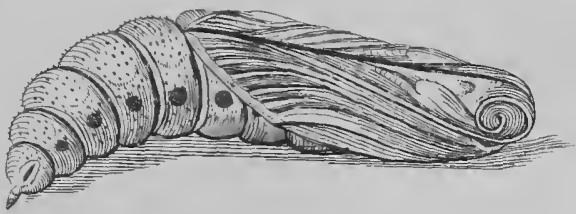

14.

I5.
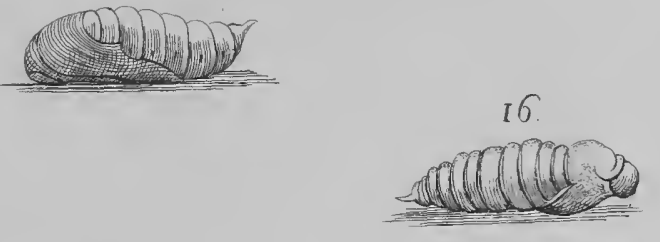

$18 . a$
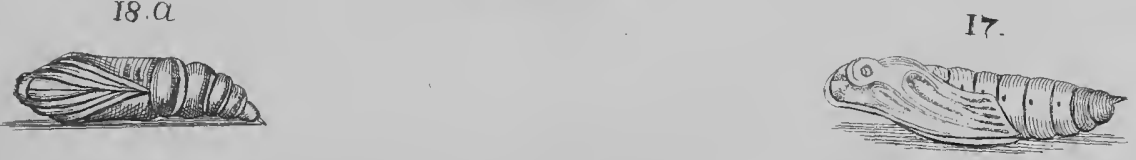

18.6
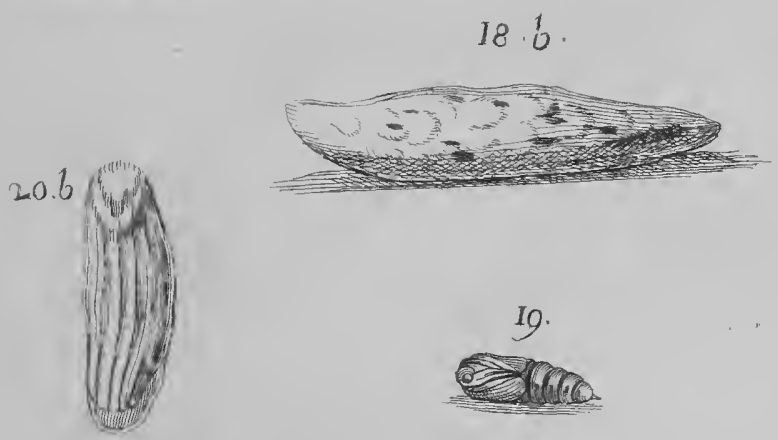

$20 . a$.
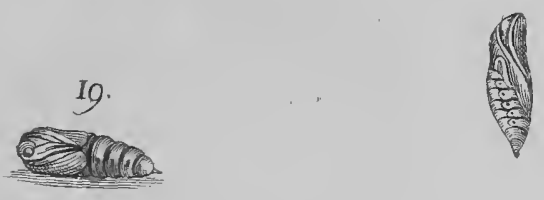



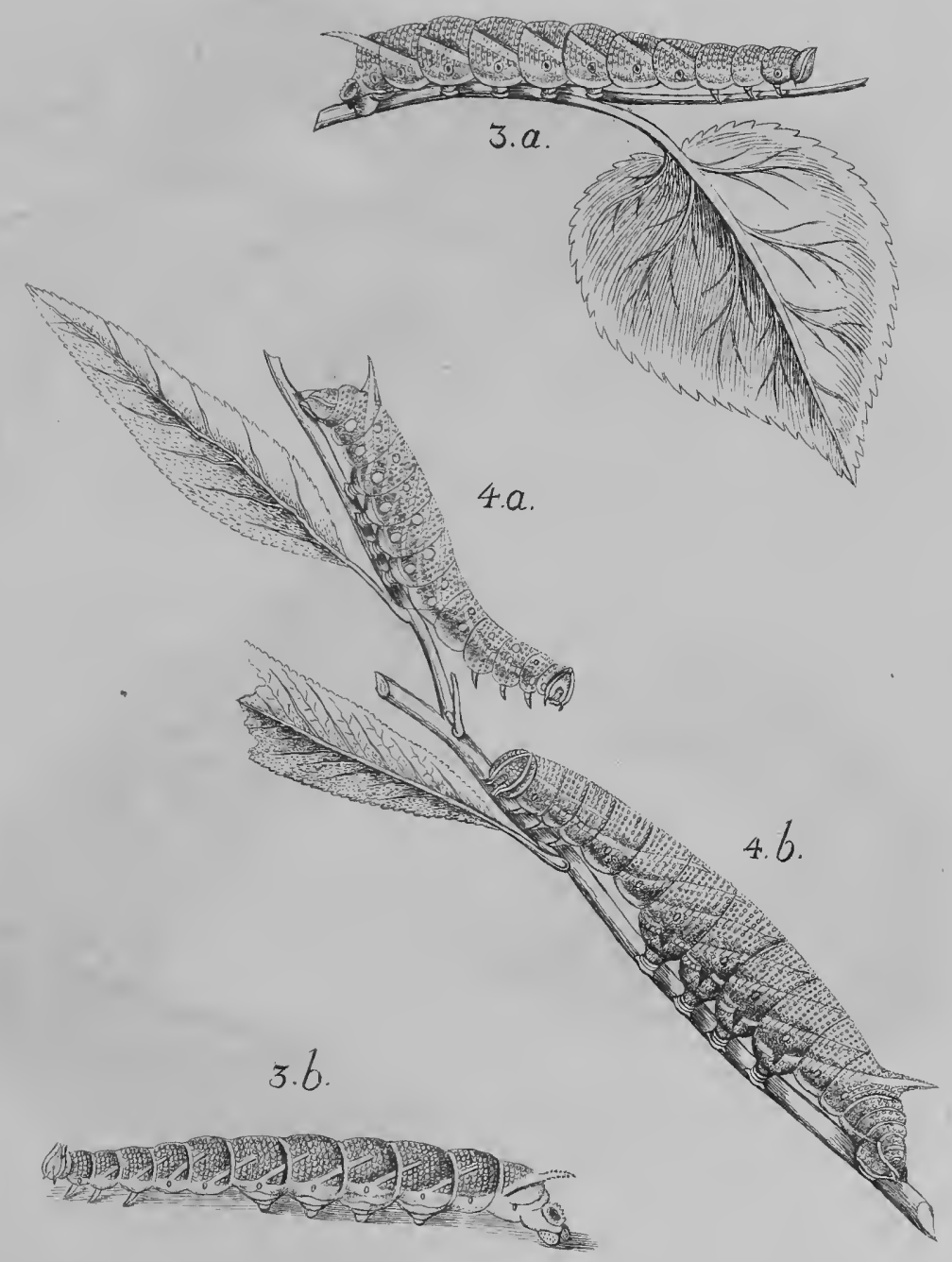




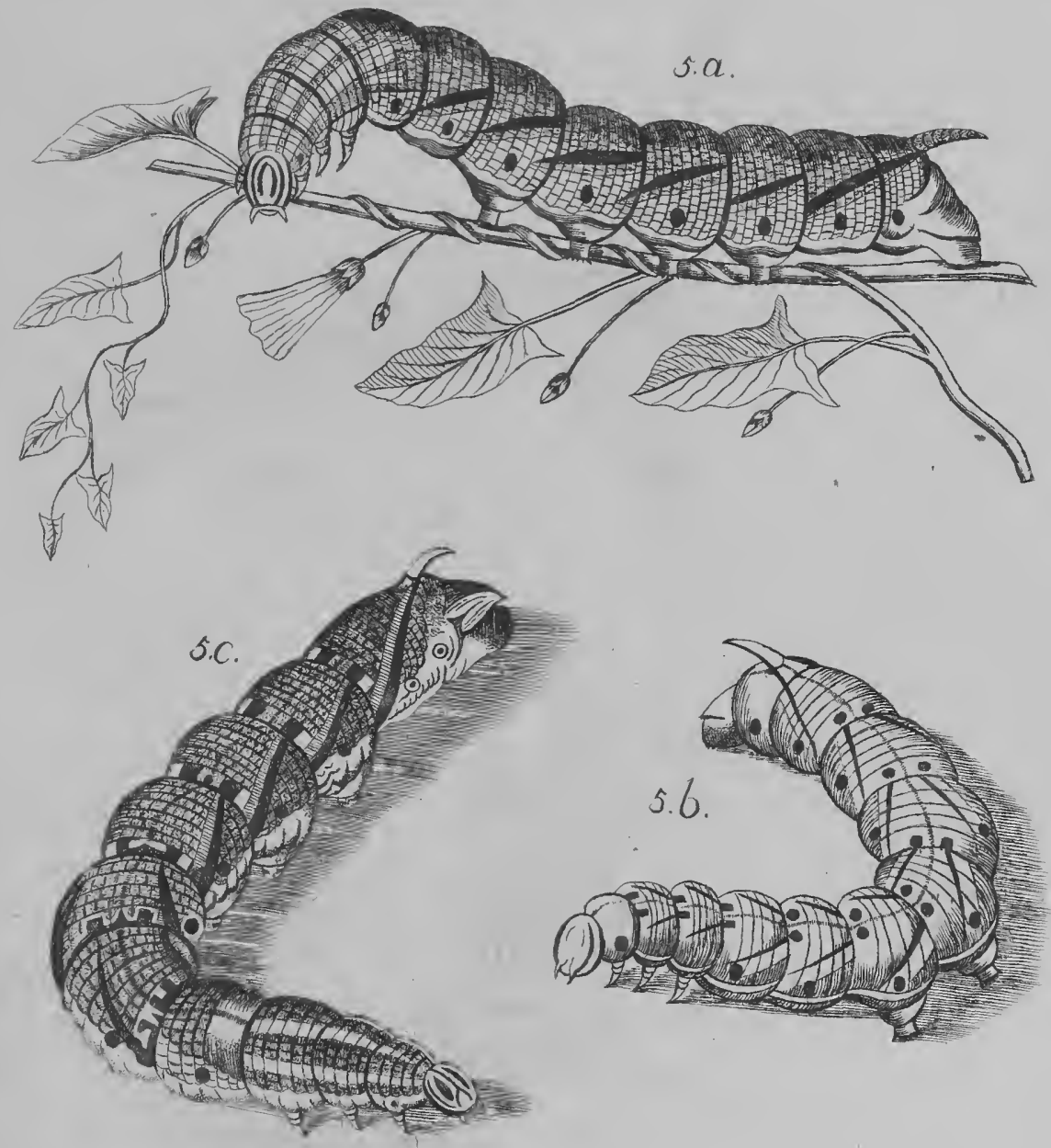




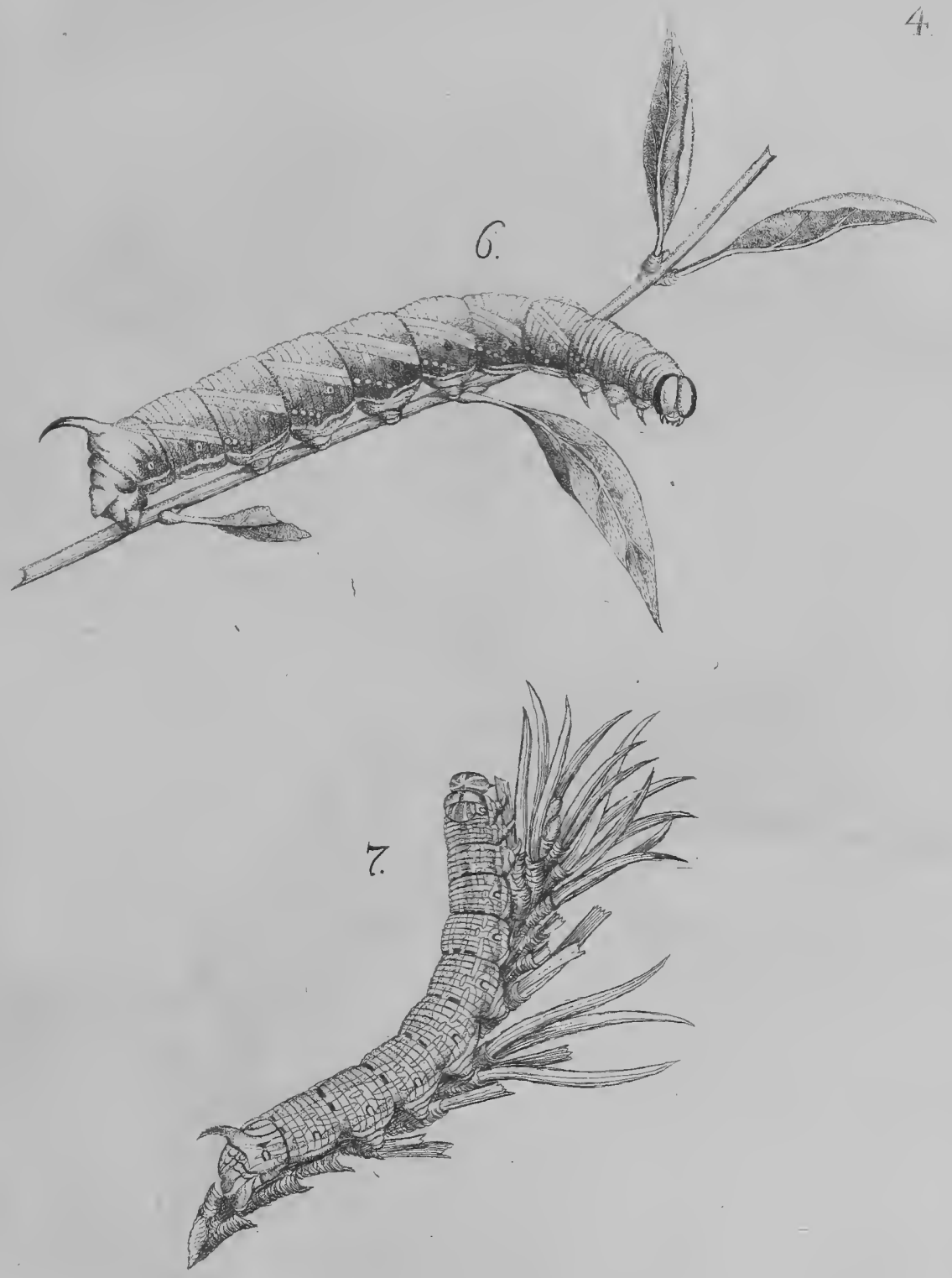



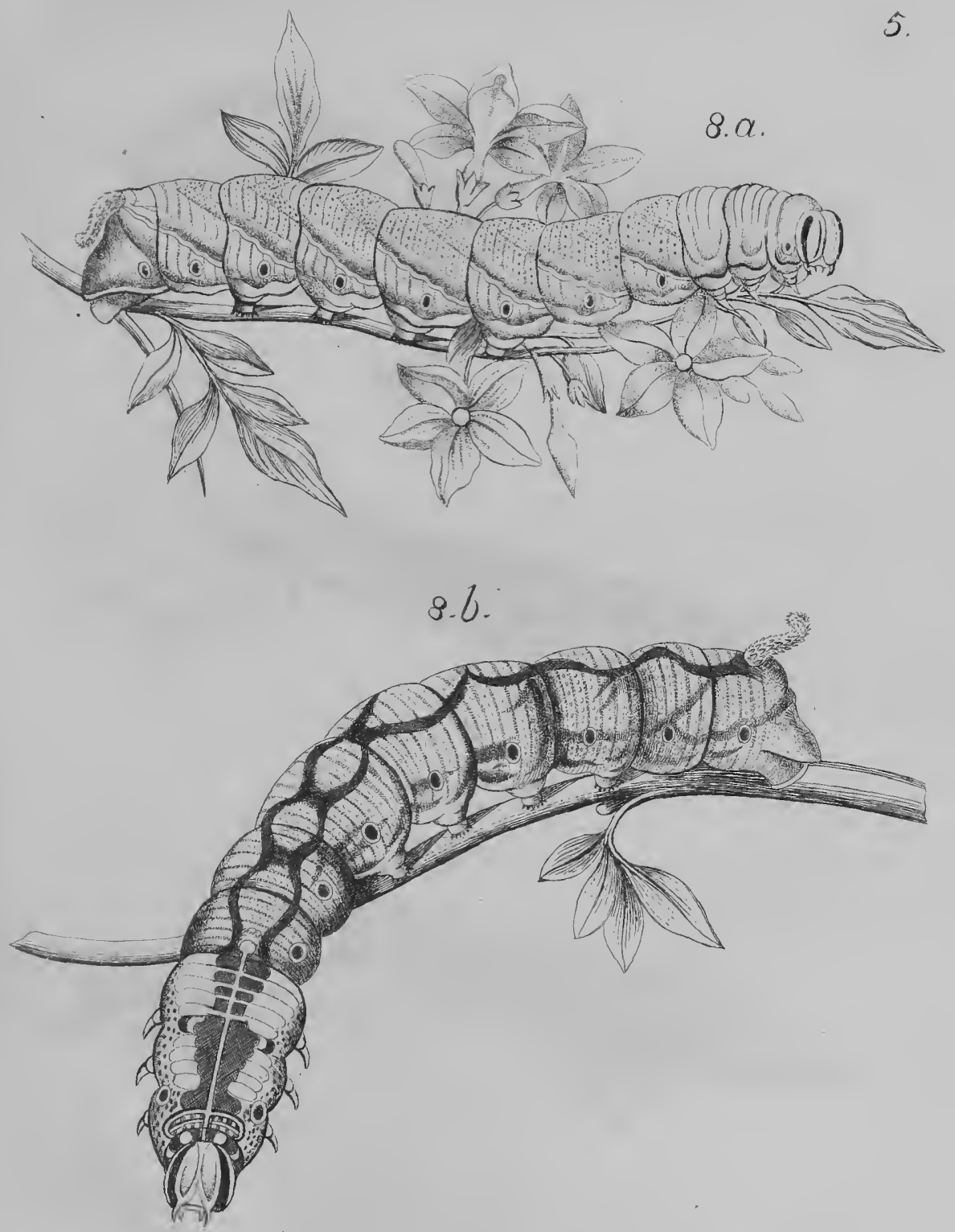


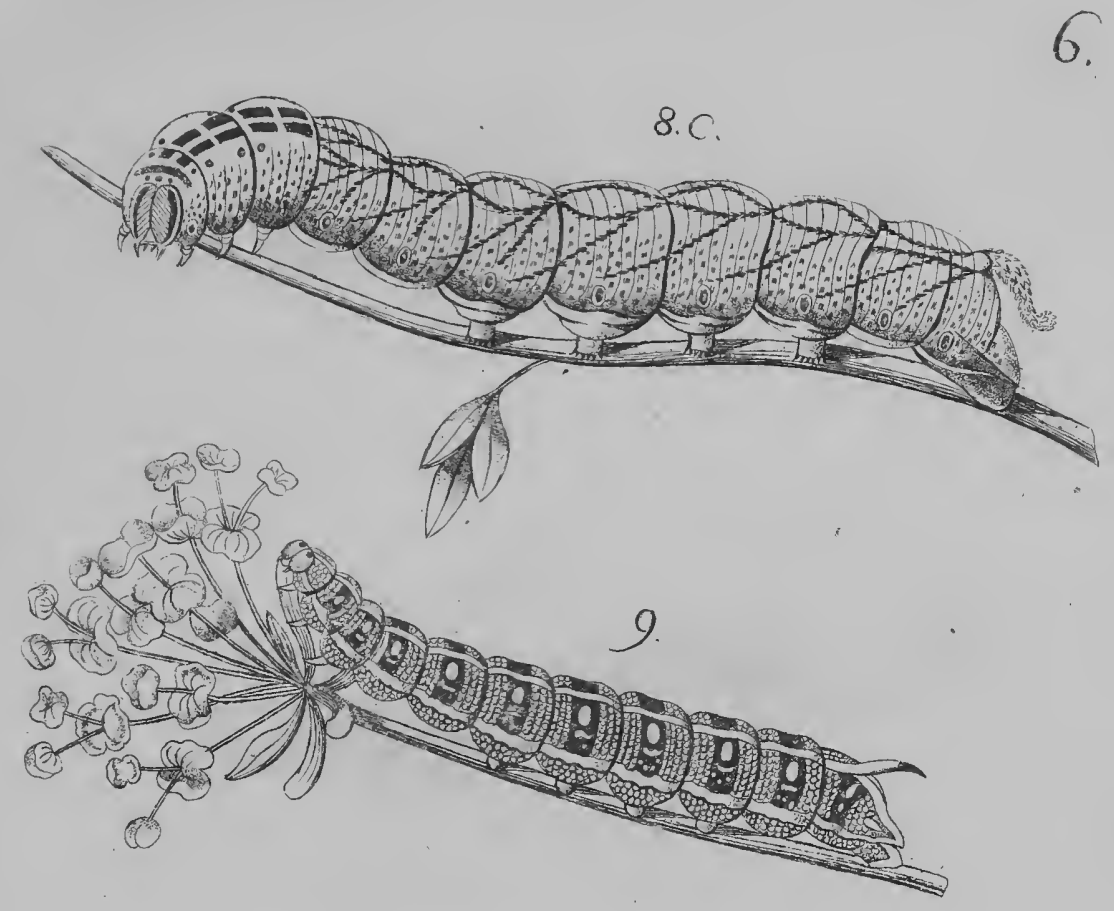

10. $a$.

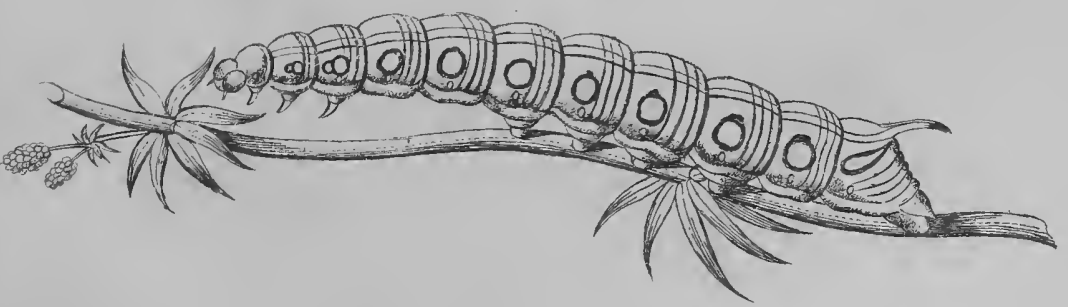


10. $b$.

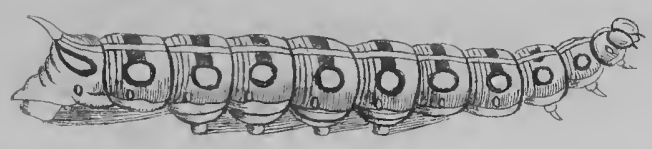

10. C .
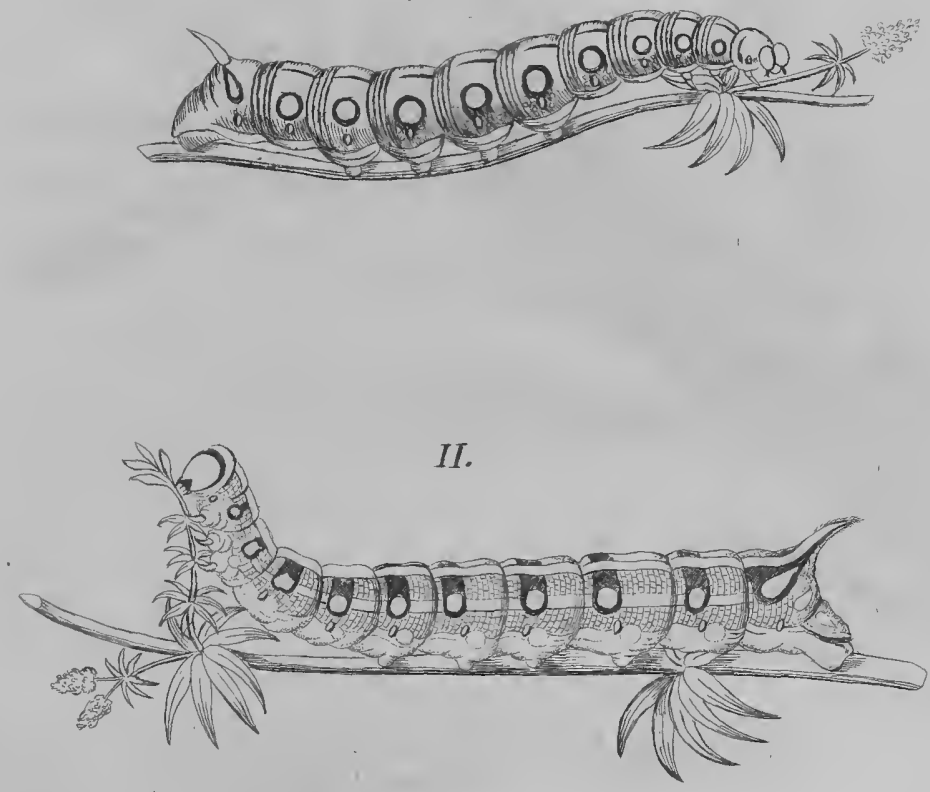
8.

\section{2. $a$.}

A.
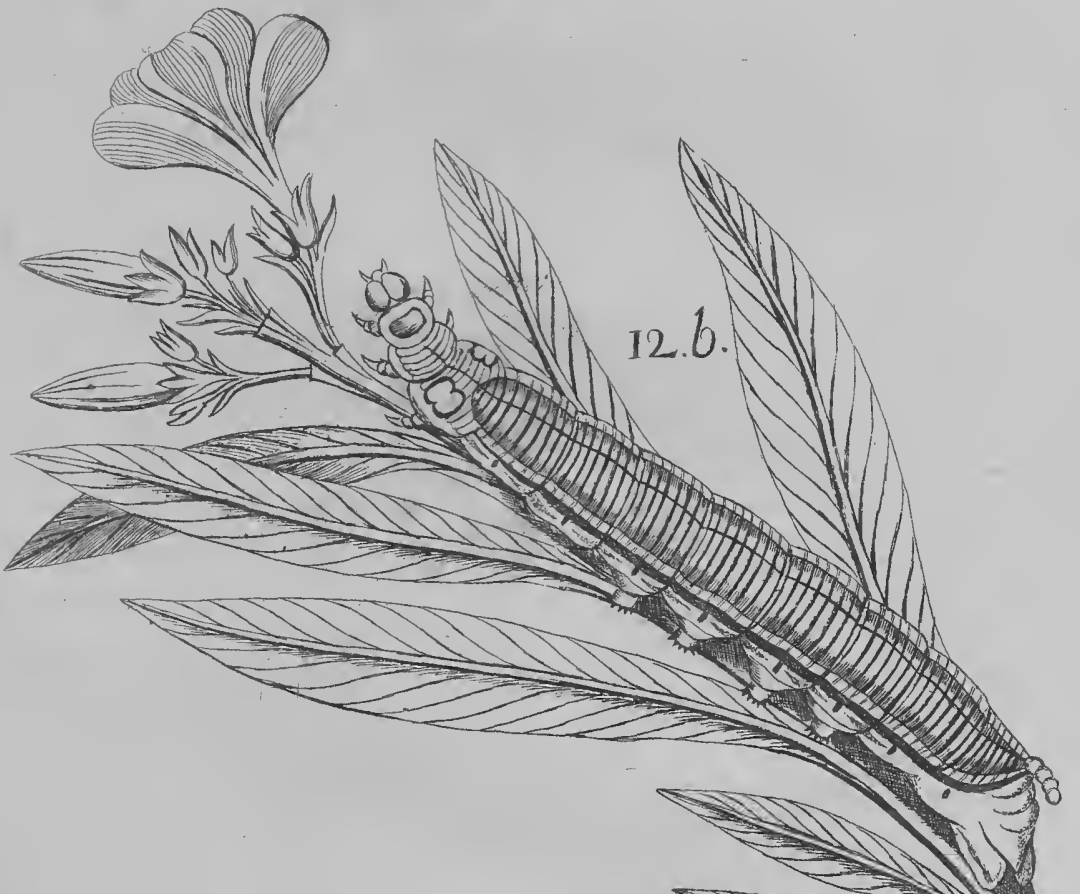

12.C.

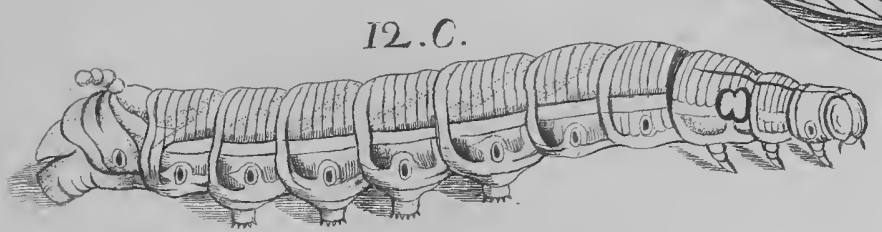

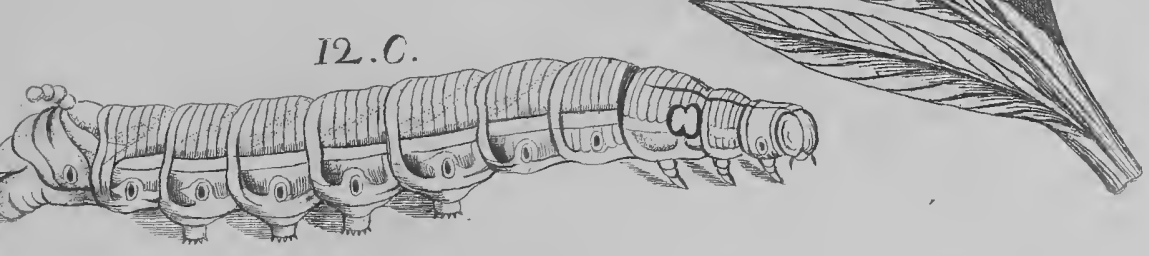



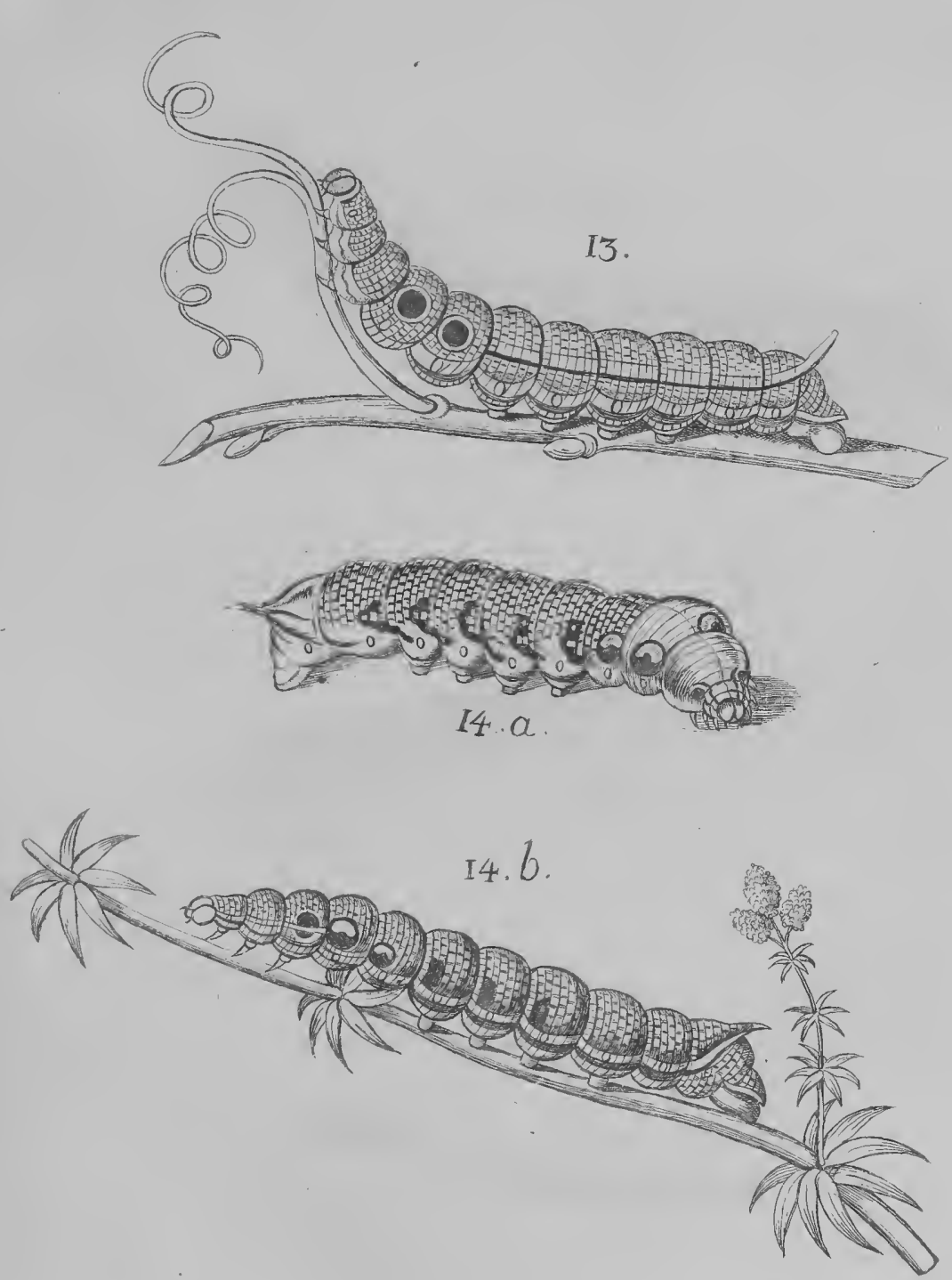
IO.
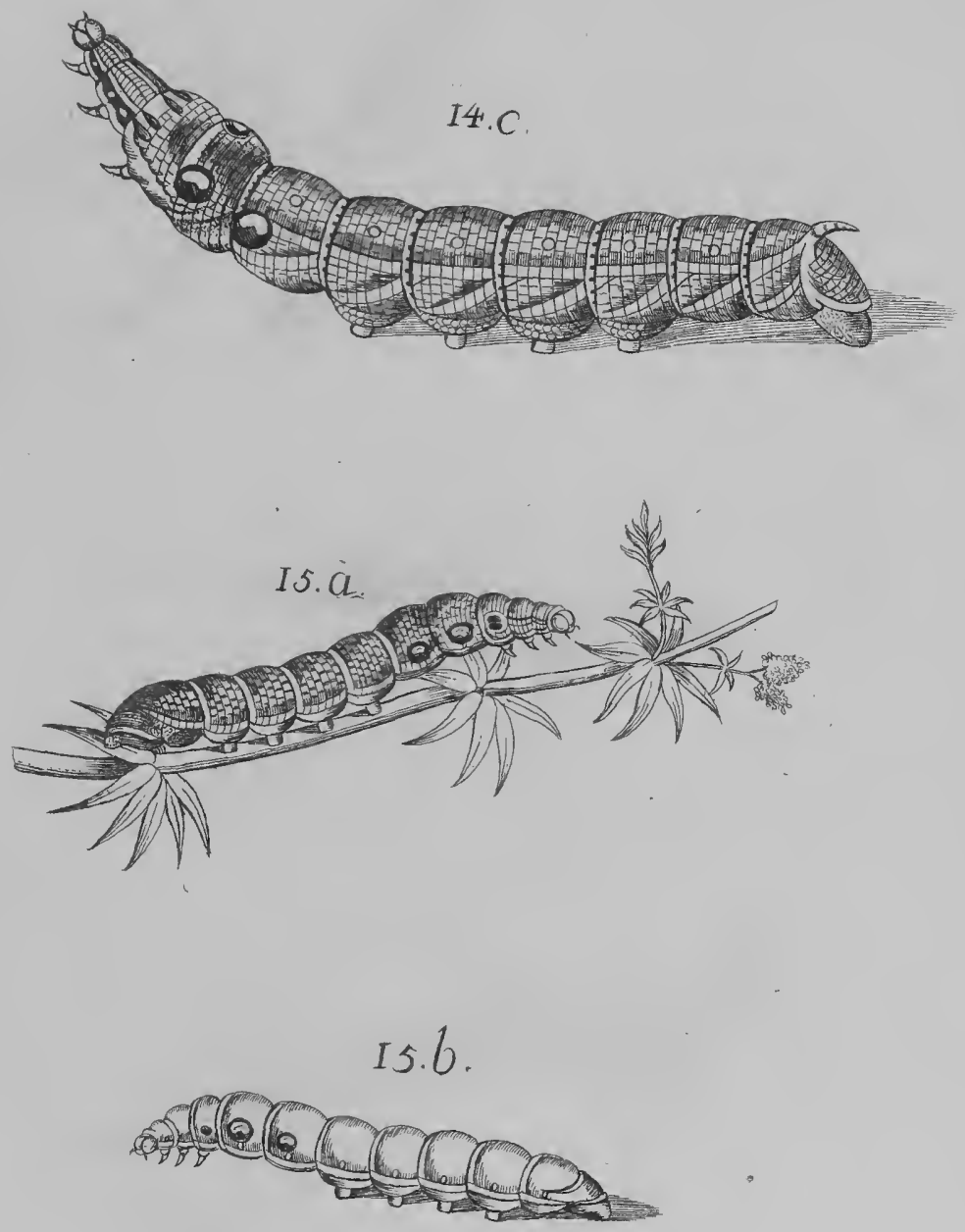


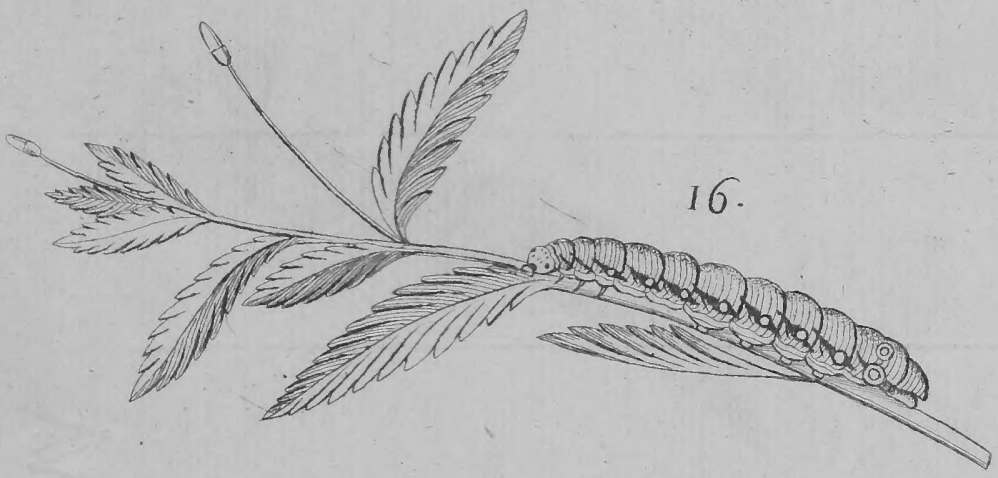

17. $a$
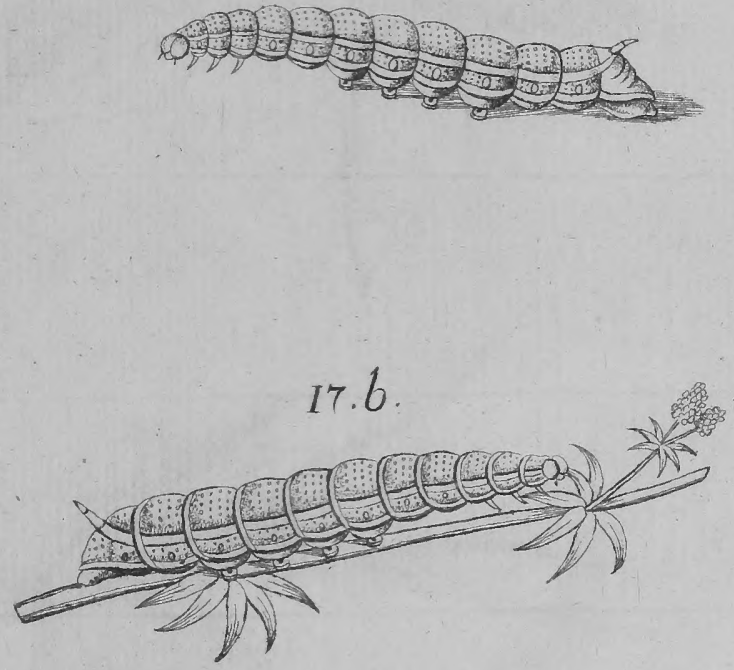

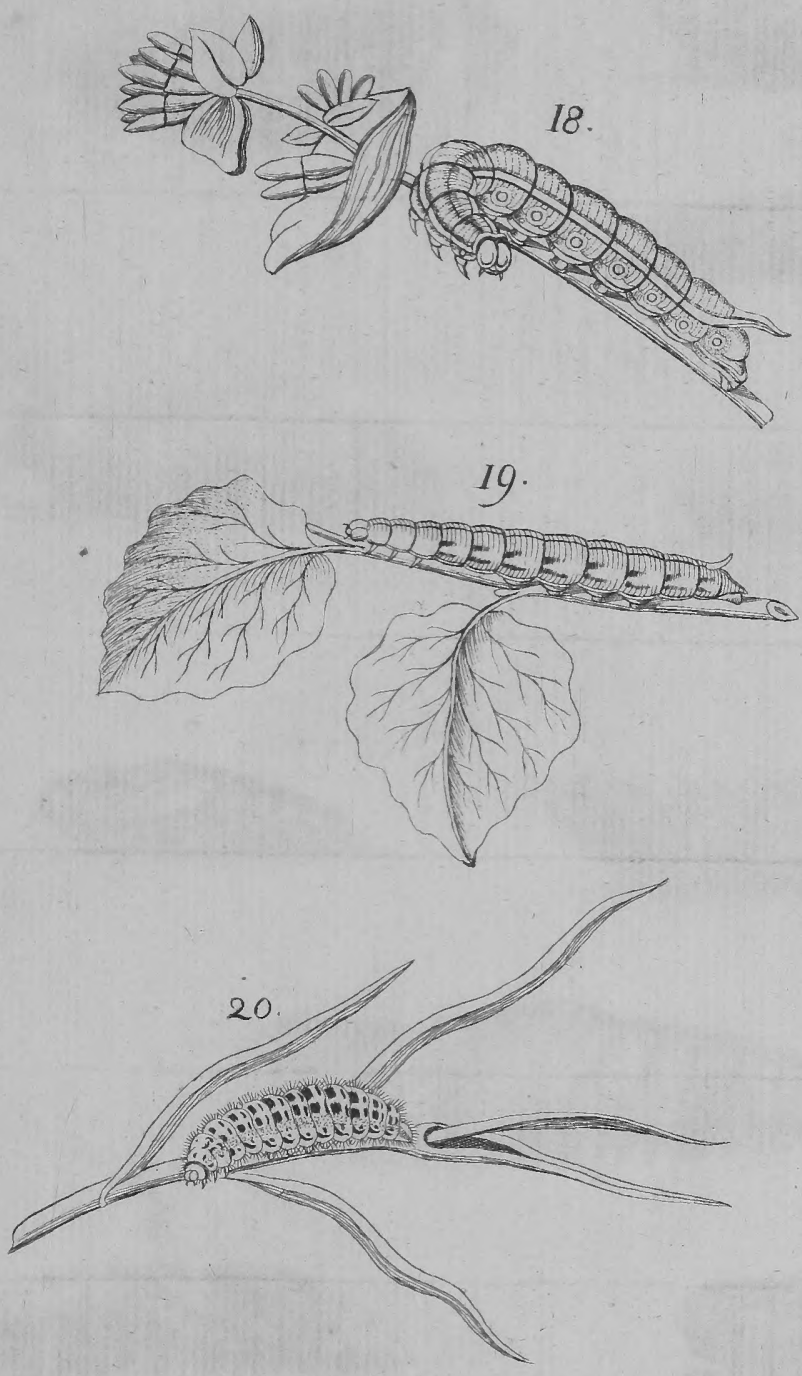
2.

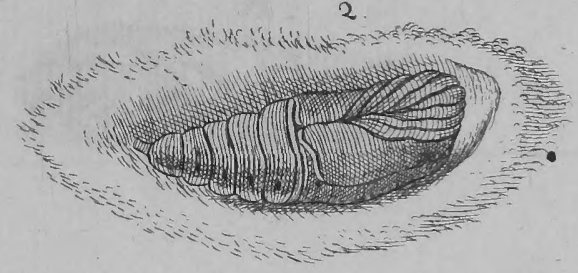

5.

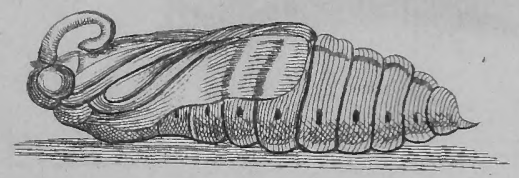

I.
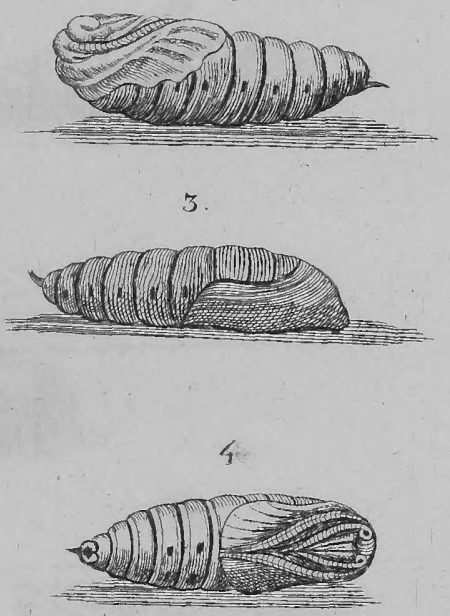
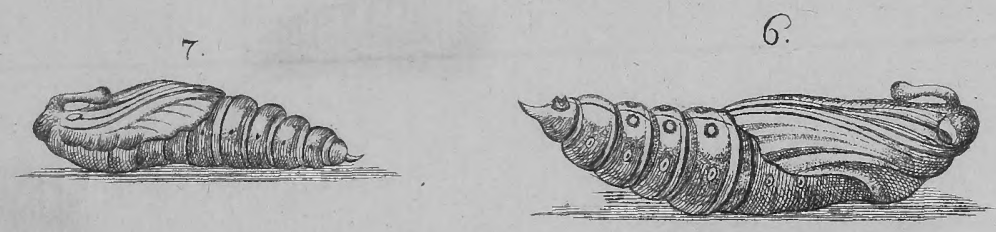

8.
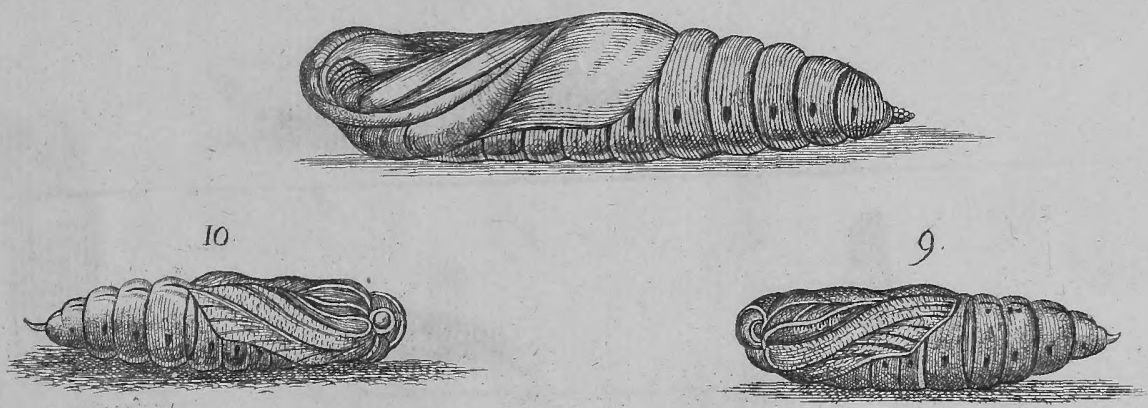
13

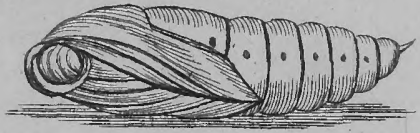

14.
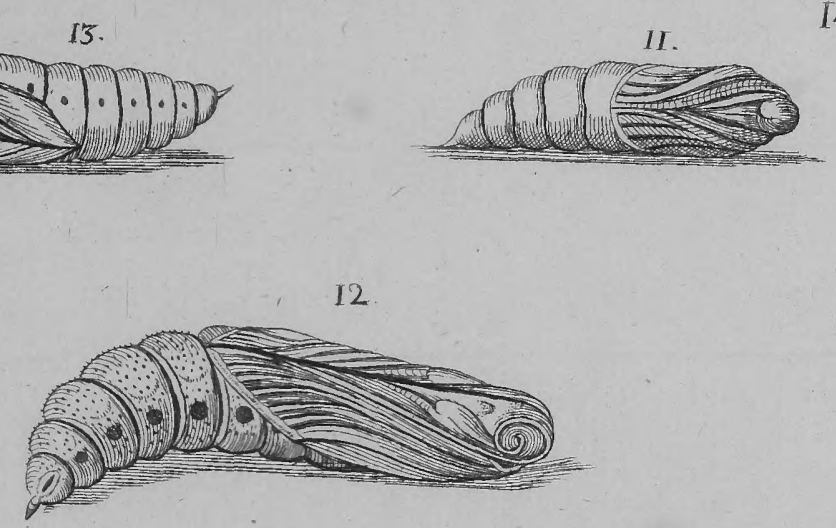

15.
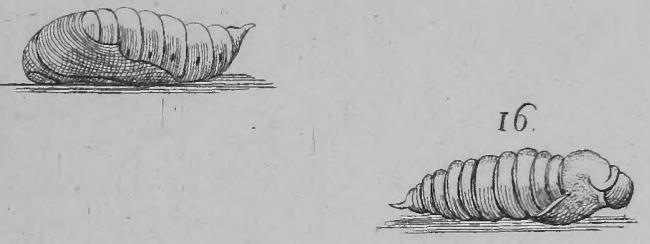

14.

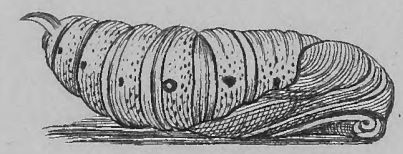

18. $a$
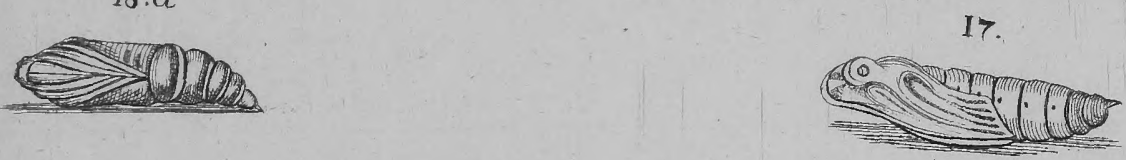

$18 \cdot 6$
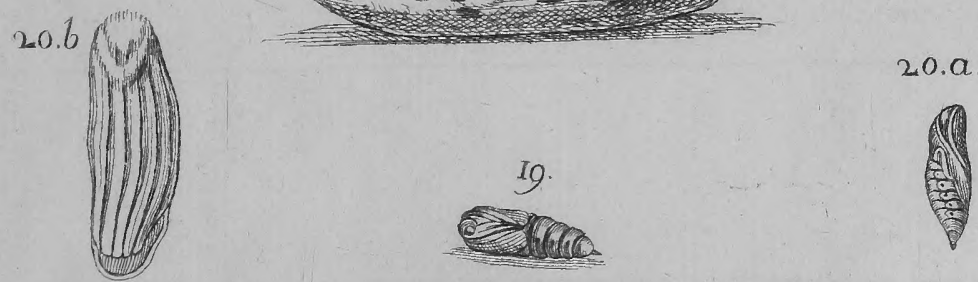